\title{
End-to-End ConvNet for Tactile Recognition using Residual Orthogonal Tiling and Pyramid Convolution Ensemble
}

\author{
Lele Cao ${ }^{1,2,3}$. Fuchun Sun ${ }^{1}$. Xiaolong Liu ${ }^{1}$. Wenbing Huang ${ }^{1}$. \\ Ramamohanarao Kotagiri ${ }^{2}$. Hongbo Li $^{1}$
}

Received: 15 April 2017 / Accepted: date

\begin{abstract}
Background and Introduction Tactile recognition enables robots identify target objects or environments from tactile sensory readings. The recent advancement of deep learning and biological tactile sensing inspire us proposing an end-to-end architecture ROTConvPCE-mv that performs tactile recognition using residual orthogonal tiling and pyramid convolution ensemble.

Methods Our approach uses stacks of raw frames and tactile flow as dual input, and incorporates the strength of multi-layer OTConvs (orthogonal tiling convolutions) organized in a residual learning paradigm. We empirically demonstrate that OTConvs have adjustable invariance capability to different input transformations such as translation, rotation, and scaling. To effectively capture multi-scale global context, a pyramid convolution structure is attached to the concatenated output of two residual OTConv pathways.

Results and Conclusions The extensive experimental evaluations show that ROTConvPCE-mv outperforms several state-of-the-art methods with a large margin
\end{abstract}

L. Cao (corresponding author)

Tel.: +4672837 7680

caolele@gmail.com, lele.cao@king.com

F. Sun, X. Liu, W. Huang and H. Li

\{fcsun, liuxl12, huangwb12, hbli\}@mail.tsinghua.edu.cn

R. Kotagiri

kotagiri@unimelb.edu.au

1. The Department of Computer Science and Technology, Tsinghua University, Beijing, 100084 China

2. The Department of Computing and Information Systems, The University of Melbourne, 3010 Victoria, Australia

3. King Digital Entertainment plc, Activision Blizzard Inc. (ATVI), 11134 Stockholm, Sweden regarding recognition accuracy, robustness, and faulttolerance. Practical suggestions and hints are summarized throughout this paper to facilitate the effective recognition using tactile sensory data.

Keywords Recognition - Tactile sensors - Feature extraction $\cdot$ Residual learning $\cdot$ Convolution neural networks $\cdot$ Tactile flow

\section{Introduction}

The fast evolving machine learning techniques and lowercost sensors are two of the many reasons that have been propelling the multidisciplinary research of robotic recognition. Robots are often equipped with multiple homogeneous or heterogeneous sensors (e.g. visual, tactile, force-torque, temperature, and position sensors) which intrinsically output sequential, redundant, and storage demanding data contaminated with noise. An efficient method assisting robotic recognition of objects and/or environments based on sensory readings could bring robots one step closer to accomplishing precise operations by, for example, adapting gripping force to the softness, stiffness, and the surface texture of the object [1]; therefore, the sensory input plays an essential role in the hotspot research areas of robotic recognition and dexterous manipulation. As an important supplement to visual inputs, tactile sensation becomes crucial in particular when the visual perception is impaired or non-discriminative.

Tactile sensor is a device for measuring spatial and temporal property of a physical contact event [2]; one tactile sensor usually contains an array of sensing units, which measure the orthonormal pressure using a predefined frequency. The output value of each sensing unit may range quite differently from one type of sensor to 
Sequential tactile readings (in 2D frames) generated in a real time manner

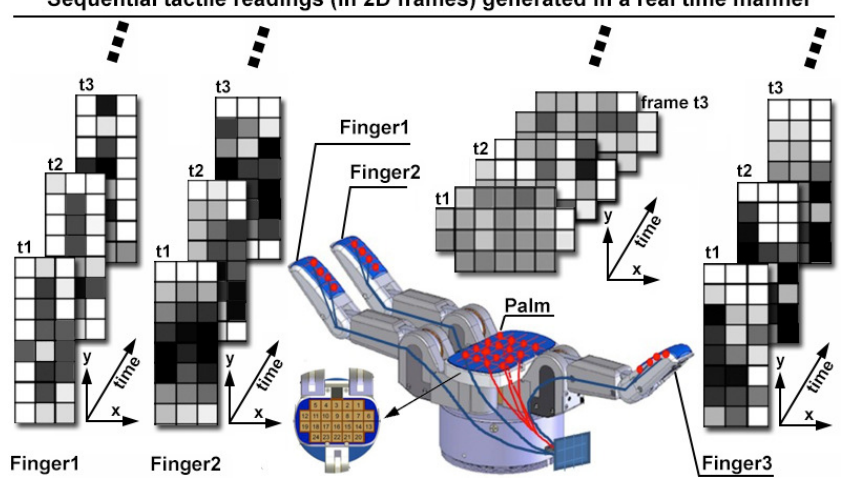

Fig. 1 An example of tactile sequences (3 fingers +1 palm) obtained from the Barrett Dexterous Hand (BDH). The alignment of finger sensor units can be referred to in Fig. 4a.

another; but if we normalize all values to gray-scale intensities (i.e. integers from 0 to 255), and visualize them as images, we will obtain sequences of grayscale "image" frames like the ones illustrated in Fig. 1, where a robotic hand with tactile sensitivity grasps an object, its tactile sensors output tactile data (a.k.a. tactile sequences), whose consecutive frames are correlated over time. Tactile data have been explored extensively in target recognition (e.g. [3-7]), material/shape awareness (e.g. [8-10]), grasp stability estimation (e.g. [11-13]), slippage detection (e.g. $[14,15]$ ), etc. In this paper, we address the problem of tactile object recognition, which aims at identifying target objects based on tactile data. For that purpose, we propose an end-to-end network that simultaneously learns the spatio-temporal tactile features and a discriminative object classifier. Our network is hereafter formally referred to as ROTConvPCEmv (residual orthogonal tiling convolution with pyramid convolution ensemble and majority voting), which has neither prior assumption on the nature of tasks nor dedicated feature extraction phase. Our approach mainly has the following advantages over previous work.

- Stacking of tactile frame and flow: spatial and temporal features are learned from the raw frames and the inter-frame flow respectively.

- Orthogonal random filter-initialization: the orthogonally initialized weights make the optimization easier and faster to converge.

- Filter-tiling convolution: it allows complex data invariances in various scales.

- Residual learning framework: it effectively prevents learning degradation and greatly reduces the work of hyper-parameter selection.

- Spatial pyramid convolution ensemble: it enables learning multi-scale global features by incorporating filters of various sizes.
- End-to-end network architecture: one unified network learns both the internal tactile representations and an object classifier directly from the raw tactile sequences.

The rest of this paper is organized as follows. In section 2 , we review the previous work of tactile recognition approaches. Section 3 gives a thorough introduction to an improved convolution operation, which is served as a fundamental building block of our proposed network (cf. section 4). The extensive experimental analysis on several tactile recognition tasks is presented in section 5; and finally the conclusions are summarized in section 6 .

\section{Related Work}

The mainstream approaches to build representation for tactile data used to follow the manual feature extraction methodologies; they emphasize mostly on spatial information and require careful selection of feature descriptors according to the properties of the data and task. The methods in this category largely fit into two categories: 1) hand-designed descriptors such as eccentricity $[16] ; 2)$ extension from existing descriptors originated in other domains such as Tactile-SIFT [17]. In the recent years, the other stream of tactile features have been proposed by applying various machine learning approaches such as principal component analysis [18] and random forests [7].

Realizing the importance of exploiting the temporal dimension, a few researchers (e.g. [19,20,8,4]) developed ways to model series of tactile data; but they still need predefined feature types or distance measures. Coincide with the biological discovery (e.g. [21]) of separate neural pathways for processing either spatial or temporal information embodied in the tactile stimuli, extracting spatial and temporal features in parallel from raw tactile data has become an active research branch. According to the phase when spatial and temporal features are fused to support meaningful predictions, any spatio-temporal tactile recognition model falls into either category of early-fusion or late-fusion. The earlyfusion approaches strive to build a spatio-temporal joint representation from raw tactile data at the very beginning, while late-fusion approaches usually construct spatial and temporal features separately, which are thereafter fused during later phases.

The early-fusion approaches embody a variety of studies on modeling time-series data. In [22], authors simply described each tactile frame with mean intensity and standard deviation, measured the temporal characteristics using pairwise distances, and applied the $\mathrm{k}$ - 
nearest neighbors algorithm to classify tactile sequences. Liu et al. [23] improved the spatial descriptor with Joint Kernel Sparse Coding (JKSC) to exploit the intrinsic relations between the touch-sensitive fingers. In [5], the technique of bag-of-features is used to create a feature vocabulary for tactile observations. The aforementioned approaches are either task-specific or sensitive to local variances. However, robotic grasping with even a tiny alteration of contact points, grip force, and initial pose could incur translation, scale, and rotation deformations respectively, to the tactile patterns for grasping the same object. To handle scale variance, Liu et al. [8] normalized strongly correlated tactile frames into a single map, from which, scale-invariant features are extracted by filtering, segmentation, and binarization. But their algorithm is combinatorially infeasible for large number of features and not invariant to translation or rotation. The authors of [6] proposed the SpatioTemporal Hierarchical Matching Pursuit (ST-HMP) to capture tactile properties for $3 \mathrm{D}$ cubes sampled from blocks of consecutive frames. ST-HMP is used by [24] in a reinforcement learning setup to achieve self-supervised re-grasping tasks. Most recently, ConvNets (Convolution Networks) have started to be used as tactile feature extractors such as in [25]. A less commonly seen approach in this league is to model series of tactile frames by means of Hidden Markov Models (HMMs) [19,11], Gaussian Process (GP) [20], and Linear Dynamic Systems (LDSs) $[4,5,26]$. Particularly, [26] has shown satisfying results by performing sparse coding and dictionary learning on LDSs. Nevertheless, we notice that all these trials directly modeling the tactile series have to manually select feature descriptors or distance measures.

On the other hand, the late-fusion approaches often adopt a multi-streamed structure, where each stream is a spatial (or temporal) feature extractor; and their outputs are further aggregated to generate predictions for specific tasks. In [6], a comparative method MVHMP that combines classification results for individual tactile frames via majority pooling was introduced; but it failed to generalize to all object classes. While there is very few late-fusion endeavors to model tactile sequences, there exist many of those in the domain of computer vision. Authors of [27] formally illustrated the late fusion model that places two single-frame ConvNets a distance of 15 frames apart and merges their pooled features in the last fully connected layer; though the last layer can compute global motion characteristics, neither single-frame stream alone can capture any local motion. Building on the research of moving object detection [28], Tu et al. [29] proposed to use global optical flow and region-based contrast as temporal and spatial feature descriptor, which are simply averaged to form a saliency map. despite of the qualitatively advantageous results by enhanced optical flow descriptors, we believe that $[28,29]$ may be further improved by utilizing auto feature extractors and nonlinear fusion strategies. Interestingly, Tnnermann et al. [30] constructed a biologically inspired two-stream saliency detection system that contains dorsal and ventral pathways, both of which encode the spatio-temporal feature using a feature template for color, symmetry, orientation, eccentricity and size; but the predefined templates limit the feature exploration capability. Simonyan et al. [31] proposed a two-stream deep ConvNets architecture which incorporates spatial and temporal networks; largely following this idea, Guo et al. [32] implemented a way to fuse visual and tactile input to aid the robotic grasping tasks.

We (some authors of this paper) recently proposed a version of late-fusion network (3T-RTCN: 3-thread randomized tiling convolutional networks) [33] for tactile recognition. In terms of overall methodological architecture, [31] is the most related work to that approach; nonetheless 3T-RTCN significantly advances and/or differentiates from theirs, since we 1) focused on tactile data that have intrinsically different physical implications from video, 2) partially untied convolutional filters, 3) removed convolutional filter-tuning, 4) adopted an efficient hierarchical fusion strategy, 5) applied majority pooling to aggregate frame labels, and 6) conducted a far more complete set of experiments on aspects such as robustness, fault-tolerance capability, and the impact of convolutional filter-tuning and late-fusion. However, all of the related work discussed so far deliberately treat feature extraction as a dedicated feature learning phase, which might in all probability diverge from how tactile stimuli is processed by human brain's cortical somatosensory areas, where tactile input is represented and recognized at the same time within somatosensory cortex areas organized in series [34]. Therefore in this paper, as an substantive extension of [33], we advances our methodologies by designing an end-to-end ConvNet using residual orthogonal tiling and pyramid convolution ensemble, which allow the tactile signal traverse through the network layerby-layer (analogous to brain's somatosensory cortex), automatically learning the hierarchical representations and recognition capabilities.

\section{Orthogonal Tiling Convolution (OTConv)}

As a variant of feedforward neural networks, ConvNets have been commonly applied to learn powerful and interpretable image features [35]. It consists of two basic 


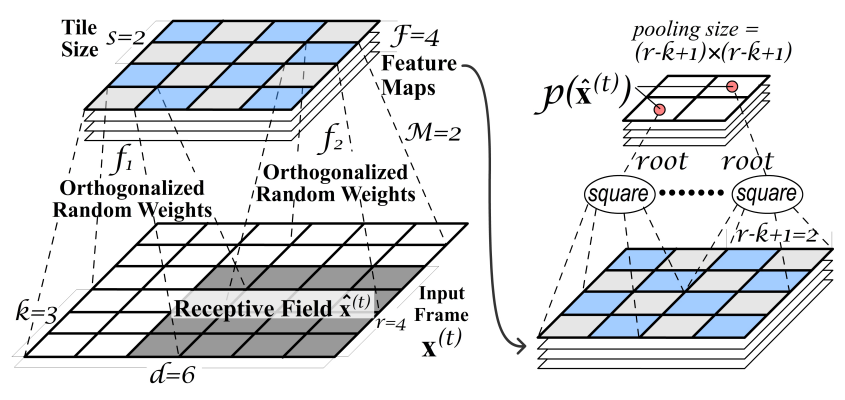

Fig. 2 A minimal example of OTConv Network; left: OTConv; right: square-root pooling. Note that only the convolution output nodes with the same color share filters.

Table 1 The formal definition of math notations.

\begin{tabular}{|c|l|}
\hline Notation & Definition \\
\hline $\mathbf{X}_{i}$ & Matrix of the $i$-th tactile sequence with $N_{i}$ frames \\
$\mathbf{x}^{(t)}$ & Vector representing the $t$-th 2D tactile frame \\
$\mathbf{y}^{(t)}$ & Vector of the ground truth class labels for $\mathbf{x}^{(t)}$ \\
$\hat{\mathbf{y}}^{(t)}$ & Vector of the predicted class labels for $\mathbf{x}^{(t)}$ \\
label $\left(\mathbf{x}^{(t)}\right)$ & The predicted object class label of the $t$-th frame \\
label $\left(\mathbf{X}_{i}\right)$ & The predicted object class label of the $i$-th sequence \\
$d$ & The dimension of $2 \mathrm{D}$ tactile frame: $d \times d$ \\
$f_{1 \ldots M}$ & $M$ different convolution filters in the same feature map \\
$s$ & Tile size: only filters that are $s$ steps away are shared \\
$k$ & Size of atom convolution area $(k \times k)$ over the input \\
$F$ & $\#$ feature maps (number of different filter sets $\left.f_{1} \ldots M\right)$ \\
$\hat{\mathbf{x}}^{(t)}$ & A local receptive field visible to one pooling output node \\
$r$ & The size $(r \times r)$ of local receptive fields \\
$p\left(\hat{\mathbf{x}}^{(t)}\right)$ & The pooling output of the local receptive field $\hat{\mathbf{x}}^{(t)}$ \\
$r-k+1$ & The size $(r-k+1) \times(r$ - $k+1)$ of pooling sub-regions \\
$n$ & The stack-size of network input $(n+1$ individual frames $)$ \\
\hline
\end{tabular}

operations (convolution and pooling) and two key concepts (local receptive fields and weight-tying). As can be seen in Fig. 2, the convolution layer may have $F \geq 2$ feature maps, so as to learn highly overcomplete representations. The pooling operations (e.g. average and max pooling) followed after the convolution operation are performed over local regions to reduce the dimensionality of features and introduce translational invariance. The concept of local receptive fields means that each pooling node only sees a small and localized area of the input; hence it ensures computational efficiency and scale-up ability. Weight-tying additionally enforces that each convolution point shares the same weights, so that the learnable parameters are reduced dramatically [36]. In this paper, we define "orthogonal tiling convolution" (OTConv) as a convolution operation utilizing filter-tiling convolution (cf. section 3.1) and orthogonal random filter-initialization (cf. section 3.2). Fig. 2 illustrates a minimal example of OTConv network containing two successive operations: OTConv and square-root pooling. The commentary of relevant math notations is formally defined in Table 1; as a generally rule, we use bold uppercase characters to denote matrices, bold lowercase characters to denote vectors.

\subsection{Filter-Tiling Convolution}

While the pooling operation inside each feature map helps to hard-code invariance to small-scale deformations [37], yet invariances to larger-scale, more global deformations like scaling and rotation might be undermined by 1) the preserved global spatial information and 2) the constraint to pool over translations of identical invariance. Gong et al. [38] addressed the first problem with multi-scale orderless pooling that extracts activations for local patches at different scale levels and performs orderless pooling over these activations at each level separately. Ngiam et al. [36] pinpointed the second problem, and developed a mechanism called filter-tiling (parametrized by a tile size noted as $s$ in Fig. 2) which leaves only convolution areas that are $s$ steps away to be shared. In terms of substance, filter-tiling has been reinvented as "dilation" (a.k.a. atrous convolution) in a semantic segmentation framework named DeepLab [39]. The filter-tiling ConvNet is expected to be capable of learning a more complex range of invariances because of pooling over convolution output nodes using different filters [36]. Since the dimensionality of tactile frames is much smaller than images in most cases, we believe that filter-tiling will provide enough flexibility in handling translation, rotation, and scale variances.

In OTConv operation, we adopt the widely used valid convolution (i.e. the filter $f$ is applied to areas on the $2 \mathrm{D}$ input where $f$ must fit completely) with zeropadding, since the authors of $[40,36]$ had verified (theoretically and experimentally) the frequency-selectiveness and the invariance quality of a similar architecture (valid convolution + square-root pooling). In the minimal OTConv network example shown in Fig. 2, for the $t$-th tactile frame $\mathbf{x}^{(t)} \in \mathbb{R}^{d \times d}$, each pooling node views a local receptive field $\hat{\mathbf{x}}^{(t)} \in \mathbb{R}^{r \times r}, r \leq d$. With tile size $s \geq 2$, we calculate each pooling node $p\left(\hat{\mathbf{x}}^{(t)}\right)$ by convolving $M$ different filters $f_{1}, f_{2}, \ldots, f_{M} \in \mathbb{R}^{k \times k}, k \leq r$ with $\hat{\mathbf{x}}^{(t)}$ :

$p\left(\hat{\mathbf{x}}^{(t)}\right)=\left[\sum_{m=1}^{M} \sum_{i, j \in L_{m}}\left(f_{m} *_{v} \hat{\mathbf{x}}^{(t)}\right)_{\langle i, j\rangle}^{2}\right]^{\frac{1}{2}}$,

where $L_{m}$ denotes the set of fields (in the form of subscription pairs $\langle i, j\rangle)$, for which filter $f_{m}$ should be applied; $*_{v}$ represents valid convolution operations. By varying $s$ (cf. section 5.3), we obtain a spectrum of models which trade off between enabling complex invariances and having few filters. A straightforward choice of $s$ would be setting it to the pooling size (i.e. $s=r-k+$ 1 ), so that each pooling node always combines untied convolution nodes. However, increasing the tile size $s$ means allowing a higher degree of freedom in the net- 
work, which might make the model more vulnerable to the risk of overfitting.

\subsection{Orthogonal Random Filter-Initialization}

Simply implementing convolution filter-tiling in ConvNets might require lengthy time to pretrain and finetune filters [27] (for sake of simplicity, we refer to pretrain and finetune as filter-tuning); this difficulty can be further compounded when the connectivity of the architecture is extended in the time-axis to enable processing multiple tactile frames at a time. We noticed several interesting results in the literature (i.e. $[41,42,40]$ ), which have shown that certain ConvNets with merely untrained random weights performed almost equally well comparing to the networks with careful unsupervised pertaining and/or discriminative finetuning. Huang et al. [43] proposed a general architecture of singlehidden-layer neural network with local receptive fields; they studied a specific implementation using orthogonalized random convolutional filters (without pretraining or finetuning) and square-root pooling, which obtained promising accuracy on an object recognition tasks and accelerated the training speed greatly. As a result, we largely follow $[36,43]$ to initialize the filter weights of OTConv layers:

Considering a certain filter $f_{m}$ in an OTConv layer, it is shared within the same feature map while distinct among different feature maps. Firstly, we randomly generate $f_{m}$ according to the standard Gaussian distribution, resulting in a weight matrix (noted as $\hat{f}_{m}^{\text {init }}$ ) of $f_{m}$ over multiple feature maps:

$\hat{f}_{m}^{\text {init }} \in \mathbb{R}^{k^{2} \times F}, m=1, \ldots, M$.

Secondly, $\hat{f}_{m}^{\text {init }}$ has to be orthogonalized to allow extracting a more complete set of features. With the concept of local receptive fields, the filters $f$ for any two convolutional nodes with non-overlapping receptive fields are already orthogonal. Moreover, Ngiam et al. [36] empirically discovered that "orthogonalizing partially overlapping receptive fields is not necessary for learning distinct, informative features". Hence, we only need to decorrelate weight matrices (i.e. columns of $\hat{f}_{m}$ ) that convolve the same region in their respective feature maps. Fortunately, this local orthogonalization is computationally cheap: the columns of $\hat{f}_{m}$ are the orthonormal basis of $\hat{f}_{m}^{\text {init }}$. To orthogonalize $\hat{f}_{m}^{\text {init }}$ for the case of $k^{2}<F$, we orthogonalize $\left(\hat{f}_{m}^{\text {init }}\right)^{\top}$ instead and then transpose it back.

As a consequence of applying filter-tiling convolution and orthogonal random filter-initialization, the OTConv may enhance the invariance ability and reduce the training time respectively; this is why OTConvs are used as the core building blocks of the our ROTConvPCEmv framework.

\section{The Proposed Approach: ROTConvPCE-mv}

ROTConvPCE-mv is the abbreviation of our newly proposed tactile recognition pipeline: Residual Orthogonal Tiling Convolution with Pyramid Convolution Ensemble and majority voting. As illustrated in Fig. 3, ROTConvPCE is an end-to-end network whose input is the interpolated stacking of individual frames and tactile flow; the two input streams are respectively feed into a 7-layer ConvNet which is organized in a residual learning framework [44]; from the concatenated outputs, multi-scale global features are then extracted using a spatial pyramid convolution ensemble. The extracted multi-scale features are respectively pooled and concatenated, and consecutively fed into a two-layer fully-connected network performing frame-level classifications. The unified sequence-level prediction is finally obtained by majority voting. In the upcoming sections where each component of ROTConvPCE-mv is introduced in a much greater detail, the tactile data are formalized as $\boldsymbol{\aleph}=\left\{\left(\mathbf{x}^{(t)}, \mathbf{y}^{(t)}\right) \mid \mathbf{x}^{(t)} \in \mathbb{R}^{d \times d}, \mathbf{y}^{(t)} \in \mathbb{R}^{l}, t=\right.$ $1, \ldots, N\}$, using the notations defined in Table 1 .

\subsection{Input: Stacking of Raw Frames and Tactile Flow}

Based on the common assumption that the distribution of static tactile force is an informative cue by itself, we stack several tactile frames together as different input channels. Specifically, for the $t$-th frame, we will take $n$ subsequent frames and stack them together with the $t$-th frame, where variable $n$ is called stack-size; so for a stack-size of $n$, there are $n+1$ individual frames staked together as input. If tactile information comes from more than one tactile sensor, many recent work (e.g. $[5,6,26])$ suggest that concatenating $2 \mathrm{D}$ readings (keeping the original layout of sensor units as is shown in Fig. 4) of multiple sensor patches is by far superior to the approaches treating sensors separately (e.g. [23]), because it allows reflecting the dependencies between multiple sensors.

The concept tactile flow was initially introduced by Bicchi et al. [45] for analyzing the tactile illusory phenomena; it is intimately related to the vision models of optical flow. Inspired by optical flow, we transform the tactile frames into grayscale images and calculate the "optical flow" (i.e. tactile flow) between adjacent "images"; we use it as a temporal feature descriptor. Since tactile readings can be hardly affected by factors 


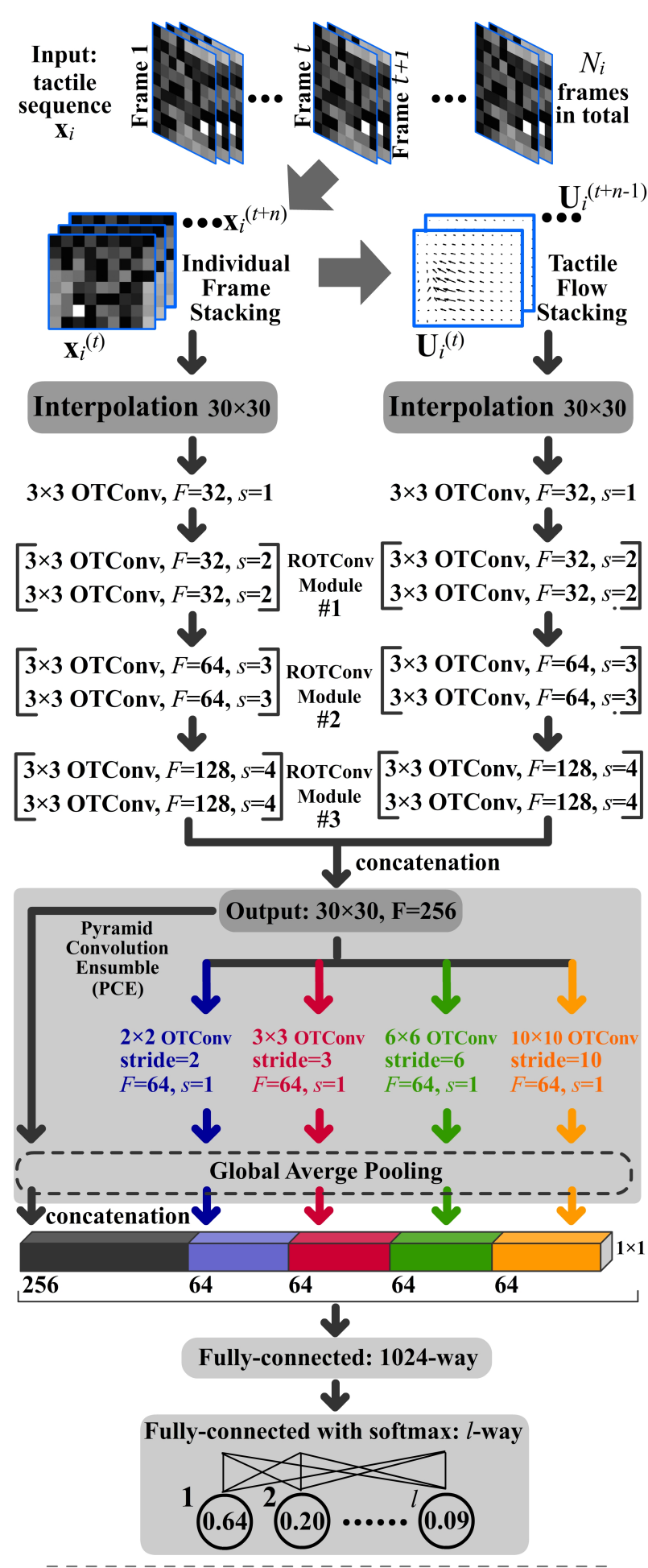

To all $N_{i}$ frames in the same tactile sequence

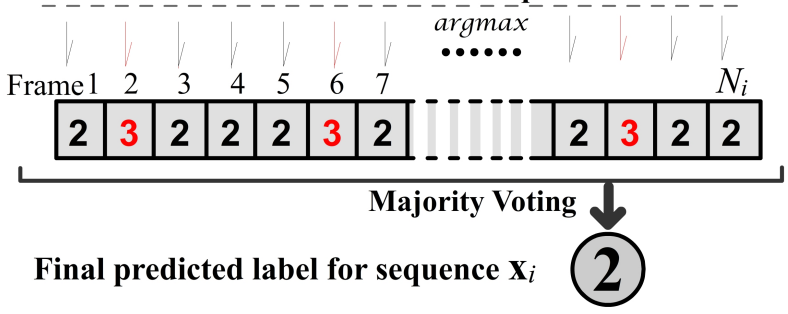

Fig. 3 The illustration of ROTConvPCE-mv framework

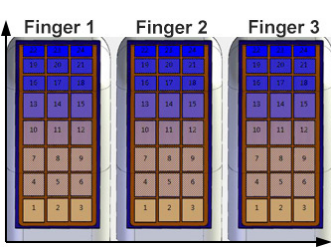

(a) Sensor unit layout

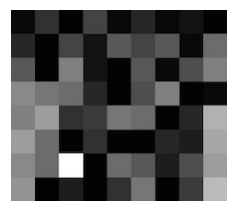

(b) Frame $t$

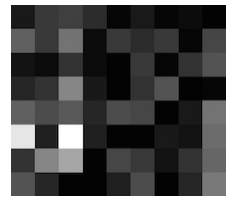

(c) Frame $t+1$
Fig. 4 An example of generating tactile frames. (a) The 3finger Barrett Dexterous Hand (BDH) has a tactile sensor patch containing $8 \times 3$ units on each fingertip; the readings from sensory units are concatenated with respect to their original spatial alignment. (b, c) The grayscale visualization ("image" with $8 \times 9$ units depicting the contact surface) of two consecutive frames in BDH-5 dataset; brighter squares stand for tactile units detecting greater normal force of pressure.

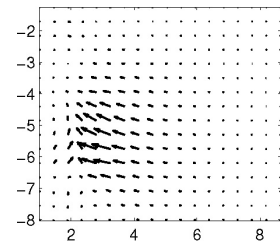

(a) Field vector

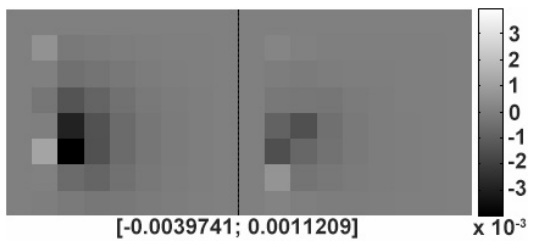

(b) Horizontal and vertical component
Fig. 5 Visualization of tactile flow between the two neighboring frames $\mathbf{x}^{(t)}$ and $\mathbf{x}^{(t+1)}$ (cf. Fig. 4b, 4c). (a) The field vector $\overrightarrow{\mathbf{u}}\left(\mathbf{x}^{(t)}\right)$. (b) The horizontal $\mathbf{u}_{\mathrm{h}}\left(\mathbf{x}^{(t)}\right)$ and vertical $\mathbf{u}_{\mathbf{v}}\left(\mathbf{x}^{(t)}\right)$ component of the vector field $\overrightarrow{\mathbf{u}}\left(\mathbf{x}^{(t)}\right)$.

like color and illumination, we empirically discover that some optical flow models addressing those factors (e.g. [46]) may have negative impact for tactile recognition and demand more computation effort. Thus we simply follow [47], and apply mean flow subtraction and tactile flow stacking.

As visualized ${ }^{1}$ in Fig. 5, we use $\overrightarrow{\mathbf{u}}\left(\mathbf{x}^{(t)}\right)$ to denote the two-dimensional vector field of tactile flow between frames $\mathbf{x}^{(t)}$ and $\mathbf{x}^{(t+1)}$; and use notation $\overrightarrow{\mathbf{u}}\left(x_{i, j}^{(t)}\right)$ to indicate the vector at point $\langle i, j\rangle_{i, j=1, \ldots, d}$. The horizontal and vertical components, $\mathbf{u}_{\mathrm{h}}\left(\mathbf{x}^{(t)}\right)$ and $\mathbf{u}_{\mathrm{v}}\left(\mathbf{x}^{(t)}\right)$, are treated as separate input channels. In our case, the vector field components can take on both positive and negative values; and the overall tactile flow can be dominated by a particular direction (e.g. caused by sudden slippage in an unstable grasping), which may be useful in slippage detection but unfavoured for ConvNets. It is normally beneficial to zero-center the input for ConvNets, as it allows better exploiting the rectification nonlinearities [31]; hence we subtract the mean vector from each vector field $\overrightarrow{\mathbf{u}}\left(\mathbf{x}^{(t)}\right)$.

Afterwards as illustrated in Fig. 3, $n$ slices of tactile flow can be calculated from the $n+1$ stacked raw tactile frames; and the stacking of those $n$ vector fields

1 For visualizing the tactile flow, we use the source code from Sun et al. [46]: http://cs.brown.edu/people/dqsun 
$\overrightarrow{\mathbf{u}}\left(\mathbf{x}^{\left(t+t^{\prime}-1\right)}\right)_{t^{\prime}=1, \ldots, n}$ represents the force motion across $n+1$ consecutive frames. The stacked tactile flow treated as the input volume $\mathbf{U}_{t}$ for an arbitrary frame $\mathbf{x}^{(t)}$ can be formulated as

$\mathbf{U}_{t}\left(i, j, 2 t^{\prime}-1\right)=u_{\mathrm{h}}\left(x_{i, j}^{t+t^{\prime}-1}\right), \mathbf{U}_{t}\left(i, j, 2 t^{\prime}\right)=u_{\mathrm{v}}\left(x_{i, j}^{t+t^{\prime}-1}\right)$,

where $\mathbf{U}_{t}\left(i, j, 2 t^{\prime}\right)_{t^{\prime}=1, \ldots, n}$ denotes the force motion at point $\langle i, j\rangle$ over a sequence of $n+1$ frames; and $\mathbf{U}_{t}$ has $2 n$ channels.

\subsection{Residual OTConv (ROTConv) Modules}

To avoid explicitly choosing the tile size $s$ for OTConv operations, we stack multiple OTConv layers with different tile sizes; by implementing such a structure, we also expect that the "levels" of features can be enriched by the number of stacked OTConv layers (i.e. depth). However, as is claimed in [44] that stacking more layers does not always ensure learning better networks; when deeper networks are able to start converging, a so-called degradation problem can emerge: with the network depth increasing, the accuracy gets saturated and even starts to decline. Therefore we integrate the residual learning [44] to OTConvs, hence obtaining the Residual OTConv (ROTConv) Modules. Fig. 6 demonstrates an example of how "ROTConv Module \#1" (cf. Fig. 3) is constructed in detail: the shortcut connection simply performs identity mapping, which is added to the output of the stacked OTConv layers. In other words, instead of hoping each few stacked layers directly fit a desired underlying mapping, we explicitly let these layers fit a residual mapping. Seen from Fig. 6, we also adopt Batch Normalization (BN) [48] after each convolution and before activation to prevent the notorious problem of vanishing/exploding gradients. We do not use more than three blocks of ROTConv modules because 1) increasing the network depth is not the only way to improve performance; there are other ways such as collecting more training data [49], applying affine data augmentation, and using filter-tilling; 2) we empirically discovered that using more than three ROTConv modules with larger tile sizes seldomly adds positive contribution to recognition rate, which probably owes to relatively lower dimensionality and less diversity of tactile frames than video frames.

\subsection{Spatial Pyramid Convolution Ensemble (PCE)}

For an usual ConvNet, the size of receptive field roughly indicates how much the larger-scale contextual information is used; for this purpose, Global Average Pooling

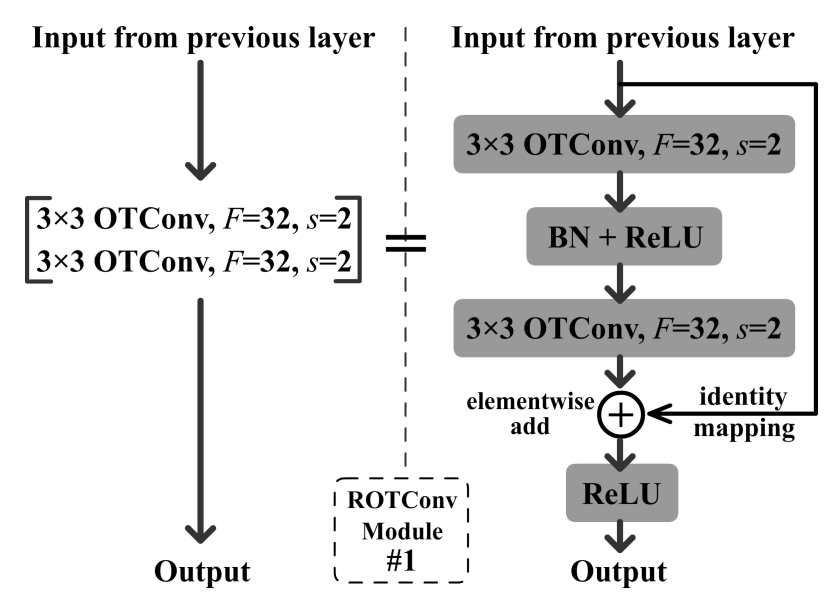

Fig. 6 The example of one ROTConv module in Fig. 3; all ROTConv modules in our network follow the same template.

(GAP) [50], as the global contextual prior, is a widely adopted baseline method in image classification networks. However, GAP is not capable of covering multiscale global information, so it tends to cause ambiguity. Recently, we have noticed that authors of [5153] have utilized Pyramid Pooling Structure (PPS) in image classification and segmentation problems, and achieved state-of-the-art results. PPS abstracts different sub-regions by applying varying-size pooling kernels to the output feature map of the last convolution layer. Intending to combine the advantages of GAP and PPS, we propose a new spatial structure of Pyramid Convolution Ensemble (PCE).

The shaded area in Fig. 3 depicts the design of our spatial PCE, which fuses features under four different OTConv scales with filter sizes of $2 \times 2$ (finest level), $3 \times 3,6 \times 6$ and $10 \times 10$ (coarsest level) respectively. By using non-overlapping convolution, the output of different levels in the pyramid convolution module contains the feature maps with varied sizes. GAP is applied to the output feature of each pyramid level, and also to the concatenated output feature from "ROTConv Module \#3" (i.e. PCE input), resulting in a GAP with a size of 512. Because the input of PCE has 256 feature maps, we use 64 filters for all OTConv operations in PCE to approximate the equal contributions between pyramid features and PCE input features.

\section{Experimental Evaluations}

The performance of ROTConvPCE-mv is extensively evaluated towards seven real-world tactile datasets with various sizes collected using different robotic platforms and experimental settings; these datasets for autonomous robot cognition tasks represent several application scenarios with different task complexities. The force values 
Table 2 Dataset specifications of the seven diversified real-world tactile datasets. Abbreviations: BDH - Barrett Dexterous Hand, SDH - Schunk Dexterous Hand, SPG - Schunk Parallel Gripper, Tf - Tactile sensor on finger, Tp - Tactile sensor on palm, Ref. - Reference(s), Seq. - Sequence(s), Avg. - Average, mani. - manipulator.

\begin{tabular}{|c|c|c|c|c|c|c|c|c|}
\hline $\begin{array}{c}\text { Dataset } \\
{[\text { Ref. }}\end{array}$ & $\begin{array}{c}\text { Hardware } \\
\text { Platform }\end{array}$ & $\begin{array}{c}\text { Sensor } \\
\text { Specification }\end{array}$ & $\begin{array}{c}\text { Per-frame } \\
\text { Dimension }\end{array}$ & $\begin{array}{c}\text { \# Object } \\
\text { Classes }\end{array}$ & $\begin{array}{c}\text { \# Total } \\
\text { Seq. }\end{array}$ & $\begin{array}{c}\text { Avg. Seq. } \\
\text { Length }\end{array}$ & $\begin{array}{c}\text { \# Train } \\
\text { Frames }\end{array}$ & $\begin{array}{c}\text { \# Test } \\
\text { Frames }\end{array}$ \\
\hline $\begin{array}{c}\text { SD-5 } \\
{[19,11]}\end{array}$ & $\begin{array}{c}\text { 3-finger SDH } \\
\text { Weiss sensor }\end{array}$ & $\begin{array}{c}\mathrm{Tf}(\times 3): 13 \times 6 \\
\mathrm{Tf}(\times 3): 14 \times 6\end{array}$ & $27 \times 18$ & 5 & 51 & $\begin{array}{c}342 \\
\text { frames })\end{array}$ & $\begin{array}{c}11,942 \\
\text { (avg. }\end{array}$ & $\begin{array}{c}5,497 \\
(\text { avg. })\end{array}$ \\
\hline $\begin{array}{c}\text { SD-10 } \\
{[19,11]}\end{array}$ & $\begin{array}{c}\text { 3-finger SDH } \\
\text { Weiss sensor }\end{array}$ & $\mathrm{Tf}(\times 3): 13 \times 6$ & $13 \times 18$ & 10 & 100 & 349 & 25,001 & 9,857 \\
\hline $\begin{array}{c}\text { SPr-10 } \\
{[22]}\end{array}$ & $\begin{array}{c}\text { 2-finger SPG } \\
\text { Flexible sensor }\end{array}$ & $\mathrm{Tf}(\times 2): 8 \times 8$ & $8 \times 16$ & 10 & 97 & 511 & 36,782 & 12,787 \\
\hline $\begin{array}{c}\text { SPr-7 } \\
{[22]}\end{array}$ & $\begin{array}{c}\text { 2-finger SPG } \\
\text { Flexible sensor }\end{array}$ & $\mathrm{Tf}(\times 2): 8 \times 8$ & $8 \times 16$ & 7 & 70 & 405 & 19,827 & 8,504 \\
\hline $\begin{array}{c}\text { BDH-10 } \\
{[4]}\end{array}$ & $\begin{array}{c}\text { 4-DoF BDH } \\
\text { 7-DoF Schunk mani. }\end{array}$ & $\begin{array}{c}\mathrm{Tf}(\times 3): 3 \times 8 \\
\mathrm{Tp}: 24\end{array}$ & $8 \times 13^{\dagger}$ & 10 & 53 & 561 & 24,253 & 5,465 \\
\hline $\begin{array}{c}\text { BDH-5 } \\
{[23]}\end{array}$ & $\begin{array}{c}\text { 4-DoF BDH } \\
\text { 7-DoF Schunk mani. }\end{array}$ & $\mathrm{Tf}(\times 3): 3 \times 8$ & $8 \times 9$ & 2 & 100 & 267 & 18,452 & 8,208 \\
\hline $\begin{array}{c}\text { HCs-10 } \\
{[14]}\end{array}$ & $\begin{array}{c}\text { Handpi-50 test stand } \\
\text { Capacitive sensor }\end{array}$ & $4 \times 4$ & $4 \times 4$ & 10 & 180 & 37 & 4,720 & 1,812 \\
\hline
\end{tabular}

* The corresponding datasets are tested on a high performance server.

$\dagger$ Eight dummy units with constant zero values are added to maintain the original spatial layout (cf. Fig. 1) of sensor units.

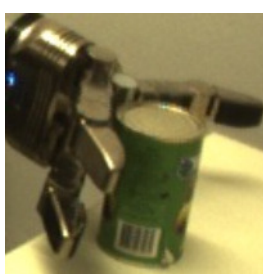

(a) $\mathrm{SDH}$

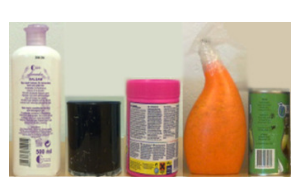

(d) SD-5

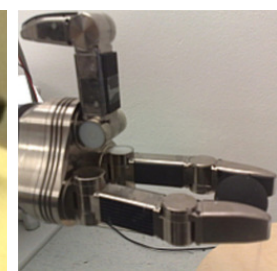

(b) 2-finger SDH

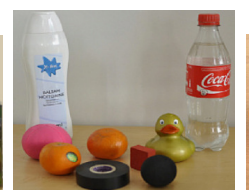

(e) SD-10, SPr-10

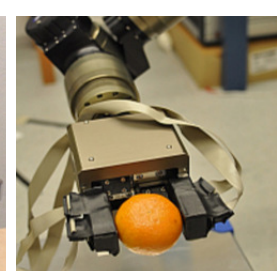

(c) SPG

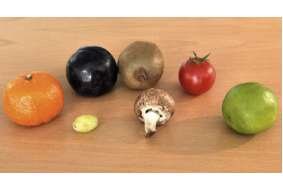

(f) $\mathrm{SPr}-7$

Fig. 7 Hardware platform and objects used to collect SD-5 $(\mathrm{a}+\mathrm{d}), \mathrm{SD}-10(\mathrm{~b}+\mathrm{e}), \mathrm{SPr}-10(\mathrm{c}+\mathrm{e})$, and SPr-7 (c+f) datasets. Figures are reproduced from $[6,11,22]$. (a) 3-finger Schunk Dextrous Hand (SDH). (b) SDH using only two fingers. (c) 2-finger Schunk Parallel Gripper (SPG). (d) Object classes in SD-5 dataset: white bottle, black cylinder, bleach cylinder, spray bottle, and can cylinder. (e) Object classes in SD-10 and SPr-10 dataset: rubber ball, balsam bottle, rubber duck, empty bottle, full bottle, bad orange, fresh orange, joggling ball, tape, and wood block. (f) 7 deformable objects for SPr-7 dataset: grape, kiwi, lime, mushroom, orange, plum, and tomato.

are normalized within $[0,1]$; and the dataset specification is shown in Table 2.

\subsection{Benchmark Datasets}

The SD-5 dataset $[11,19]$ was collected with the 3 -finger Schunk Dextrous Hand (SDH) equipped with 2 tactile
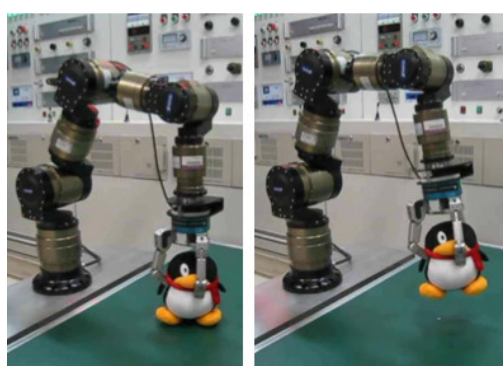

(a) 4-DoF BDH +7 -DoF Schunk mani.

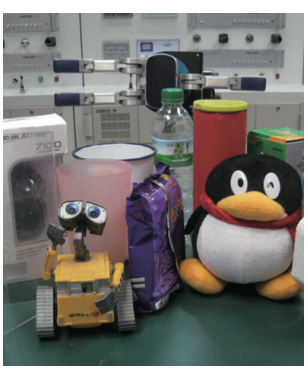

(b) BDH-10 objects
Fig. 8 The hardware platform and objects used for collecting BDH-10 dataset. Figures are reproduced from [4]. (a) Start state (left) and end state (right) of a valid grasping process. (b) 10 object classes for BDH-10 dataset: water bottle, plastic cup, enamel cup, plush penguin, plastic walle, snack bag, red cylinder, name plate, mouse box, and empty box.

sensor patches on each finger. An exemplary grasping execution of this hand is presented in Fig. 7a. As presented in Fig. 7d, there are five daily objects used for grasping. Each grasp execution provides a sequence of tactile sensor readings that is annotated with the name of the corresponding object to which the grasping was applied. The sequence consists of tactile measurements from the first physical contact with the object until the fully-closed hand configuration is reached or no more changes in tactile readings occur for a specified period of time. Each object was rotated after it is lifted; and their initial positions were not precisely aligned with respect to the hand [6]. For SD-10, SPr-10, and SPr-7 datasets $[19,11,22]$, the grasp execution applied was similar: an object was manually placed between the fingers at the beginning; after the first physical contact, the fingers 


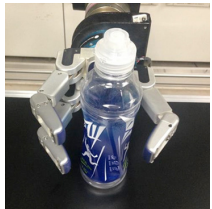

(a) Grasp pose

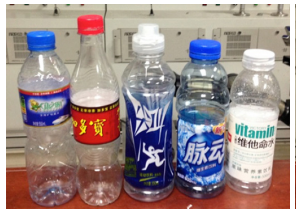

(b) Empty bottles

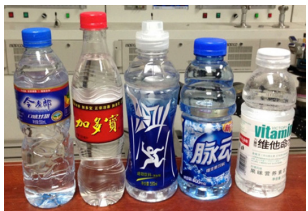

(c) Full bottles

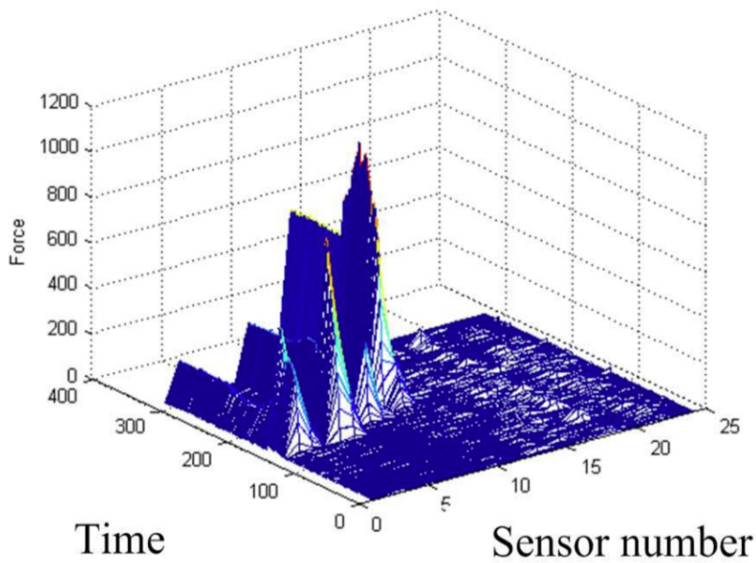

(d) Tactile readings: Empty

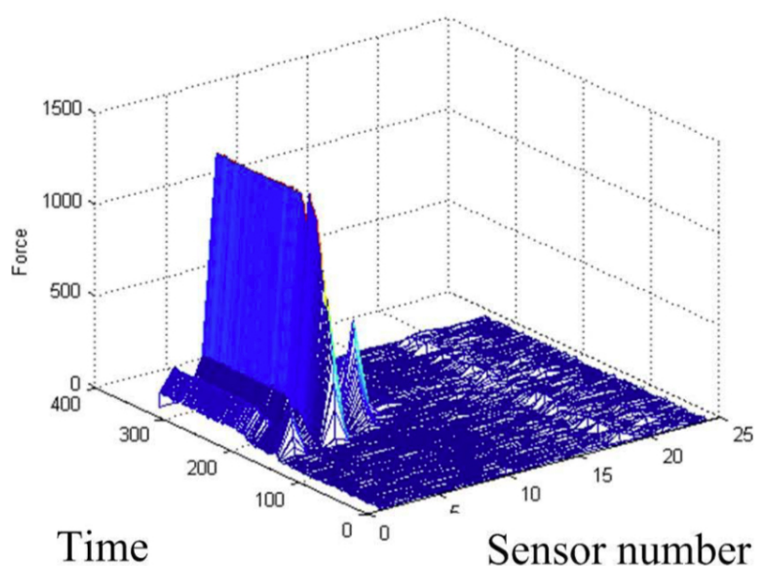

(e) Tactile readings: Full

Fig. 9 The setup for obtaining the BDH-5 dataset. (a) Grip pose of the hand. (b) Five bottles without water. (c) The same five bottles filled with water. (d) and (e) The visualization of one tactile sequence for the same bottle in empty and full status respectively.

moved back and forth slightly for five times, and then released the object in the end. The SD-10 dataset was collected by grasping ten household objects (Fig. 7e) using SDH patched with one Weiss sensor [22] on each finger (cf. Fig. 7b). The SPr-10 dataset utilized exactly the same object classes as SD-10, but was recorded from the 2-finger SPG with one $8 \times 8$ flexible sensor [22] per finger. The SPr-7 dataset used SPG in Fig. 7c to grasp 7 deformable objects in Fig. $7 f$.
The datasets BDH-10 [4] and BDH-5 [23] were collected on a dexterous robotic operation platform to perform grasping tasks (Fig. 8a and 9a). The platform is equipped with a 4 -degree-of-freedom (4-DoF) $\mathrm{BDH}$ mounted on the end of a 7-DoF Schunk manipulator. The BDH has three fingers, two of which can rotate synchronously and symmetrically around the base joint in a finger spreading action. There is one sensor patch mounted on each finger and one on the palm. Xiao et al. [4] originally created BDH-10 to help the robotic hand choosing a proper grip gesture and force by distinguishing the category of the target objects. Tactile data is recorded for every valid grasping under different placement of objects; a valid grasping process starts from the initial object contact, and ends when the lifting movement ceases (cf. Fig. 8a). Ten objects with different shape, hardness, and surface texture are grasped for $5 \sim 6$ times each, bringing about 53 tactile sequences in total. The BDH- 5 dataset was obtained by grasping 5 plastic bottles filled with water (Fig. 9c) or without water (Fig. 9b), whose visual appearances are similar and non-discriminative. Though it is hard to distinguish the two cases by visual appearance, their tactile sequences are rather different (cf. Fig. 9d and 9e). Each bottle was grasped for 10 times in a standing position; and each grasp lasts $8.2 \sim 18$ seconds. The sampling frequency is $25 \mathrm{~Hz}$, and therefore the length of the obtained sequence is $205 \sim 450$ frames. In Fig. 9a we show the representative grasping pose of the hand. From such a setting, we managed to construct a ten-object dataset containing 100 samples of tactile sequences.

Up till nowadays, there have been two typical designs in flexible capacitive tactile sensor; and the difference between them mainly lies in the selection of dielectric layer: one uses air gap, while the other uses Polydimethylsiloxane (PDMS). In [54,55], the PDMS dielectric layer is improved by the micro needle structure, therefore inheriting and synthesizing both the advantages of the capacitive sensors with air gap and that with PDMS layers. The HCs-10 dataset $[14,33]$ was gathered from this new type of capacitive tactile sensor array, several prototypes of which were recently made by Zhang et al. [55]. The single cell size in the fabricated $4 \times 4$ sensors array is $0.7 \times 0.5 \mathrm{~cm}^{2}$. As presented in Fig. 10c, we tested 10 objects with different surface properties, such as wood, metal, cloth, plastic, and foam. In order to make the objects exerting exactly the same force against the capacitive sensor, we used Handpi electronic test stand with a dynamometer mounted on a docking shelf (Fig. 10a), which can move vertically with an adjustable speed. The object was fastened at the tail of the force gauge with a clamp and is moved down towards the sensor with a small 


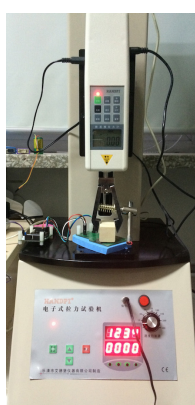

(a) Handpi-50

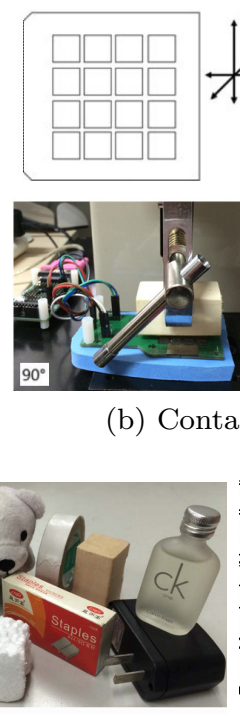

(c) HCs-10 objects
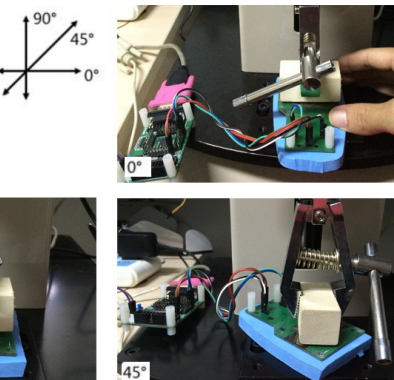

$45^{\circ}$

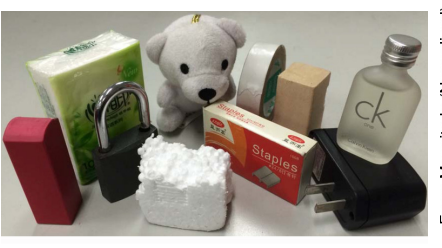

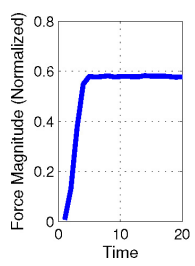

(d) Stable

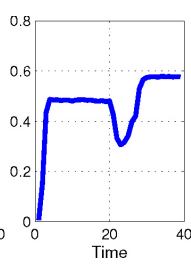

(e) Slippage
Fig. 10 An illustration of collecting the HCs-10 dataset. (a) The Handpi-50 electronic test stand with a dynamometer installed. (b) Demonstration of three directions (i.e. $0^{\circ}, 90^{\circ}$, and $45^{\circ}$ ) of object placement. (c) Ten objects in HCs-10 dataset: cf. caption of Fig. 15. (d) Single unit output in case of a stable contact. (e) Single unit output in case of a slippage right after the contact.

fixed speed. The data was logged from the initial contact until the dynamometer stopped pressing the sensor, when normal force measured by the dynamometer reached exactly 5 Newton. We placed each object in three directions as shown in Fig. 10b; and we repeated 3 times for each direction, resulting in 9 sequences for each object. To make the task more challenging, for each object, we conducted another 9 tests with the same configurations; but as soon as the dynamometer stopped, a horizontal force $P$ was applied in the same object direction to cause an object slippage. We hence have 18 tactile sequences ( 9 stable +9 slippage) collected for each object class. From the perspective of a single tactile unit, a trough of the force value often occurs in case of object slippage (e.g. Fig. 10e), which a stable contact (e.g. Fig. 10d) does not have.

To conclude, the seven selected benchmark datasets are collected using four different tactile sensors, three different robotic hands, and one force test stand; they represent eight kinds of hand-object interactions: grasping, lifting, rotating, translation, putting-down, releasing, pressing, and sliding. Their distributions along the scale of the number of sensor units, object classes, and total frame samples are summarized in Fig. 11.
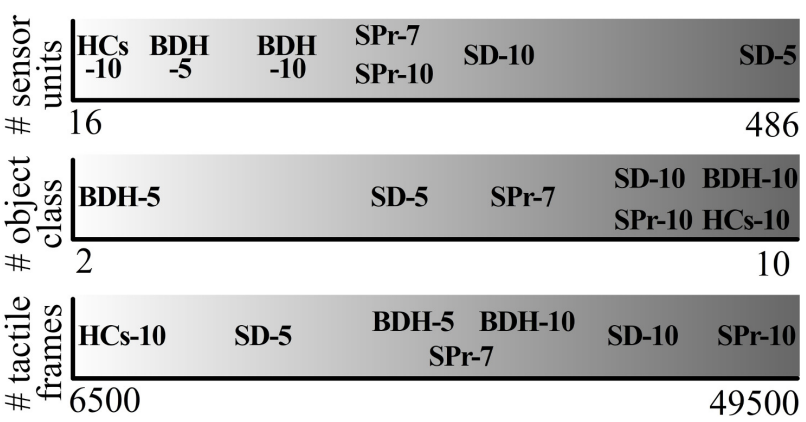

Fig. 11 Dataset distributions along the scale of the number of sensor units, object classes, and total frame samples.

\subsection{Parameter Settings and Implementation}

Largely following [44], we use BN [48] and rectified linear unit (ReLU) [56] after every OTConv operation in our network architecture. We initialize the OTConv weights as is described in section 3 and train the entire network from scratch using 10 epochs. Comparing to models like 3T-RTCN [33], ROTConvPCE-mv has helped dramatically reducing the hyper-parameter search space because 1$)$ the tile size $(s)$ and number of convolution filters $(F)$ have different values defined in different ROTConv modules, making learning the optimal combination of those parameters possible; 2) the potential advantage of combining various size of convolution filters $(k)$ is acquired by using different $k$ values in PCE branches; 3 ) by using GAP, there is no more need to select the size of pooling kernel, making the size of receptive fields $(r)$ determinate in each branch of PCE.

By carrying out a 10-fold cross-validations (9:1 splits) on the training dataset, we adopt the stochastic gradient descent (SGD) with a batch size of 64 . The effective learning rate follows a polynomial decay: $a\left(1-\frac{b}{c}\right)^{0.9}$, where 0.9 is the momentum factor; $a$ is the base learning rate $(a=0.005)$; $b$ denotes the number of elapsed training iterations; and $c$ represents the maximum number of iterations allowed (i.e. the total number of mini-batches allowed over 10 epochs). These model hyper-parameters were selected using a simple grid search approach with candidates value of batch size $\in\{16,64,128,512\}$, base learning rate $\in\{0.01,0.005,0.001,0.0005\}$, and momentum factor $\in\{0.3,0.5,0.7,0.9\})$. Subject to the optimal hyper-parameter combinations, the empirical selection of input stack-size $n$ is further discussed in section 5.4.

ROTConvPCE-mv is implemented on top of Caffe [57]. A few large datasets (i.e. the ones marked with an asterisk in Table 2) require a stationary server with high memory capacity, so we deployed those tasks on a highperformance server with dual Xeon E5-2630 2.4GHz CPU and 128G RAM. The experiments on smaller scaled 


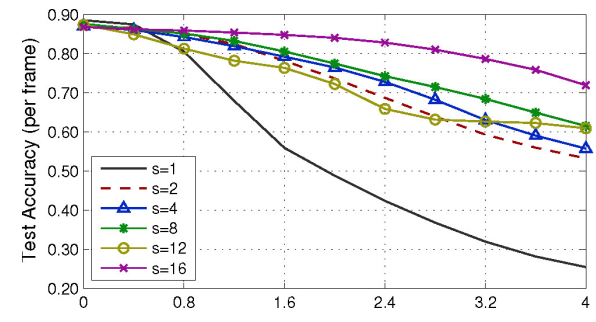

(a) SD-5: Translation (units)

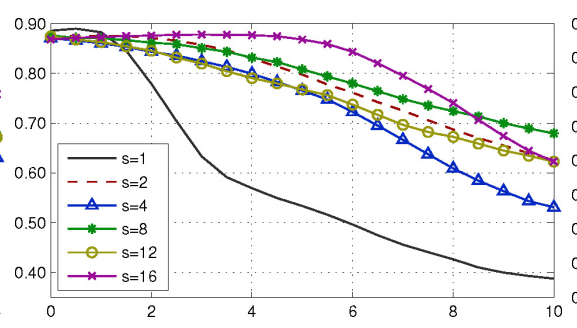

(b) SD-5: Rotation (degrees)

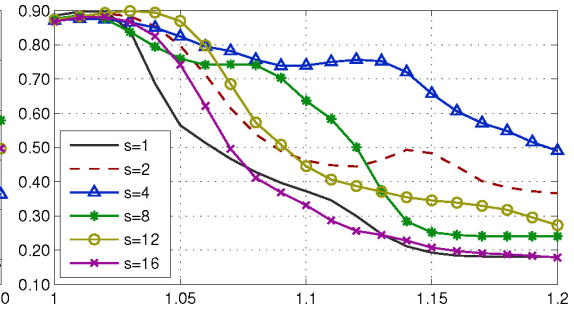

(c) SD-5: Scaling (ratio)

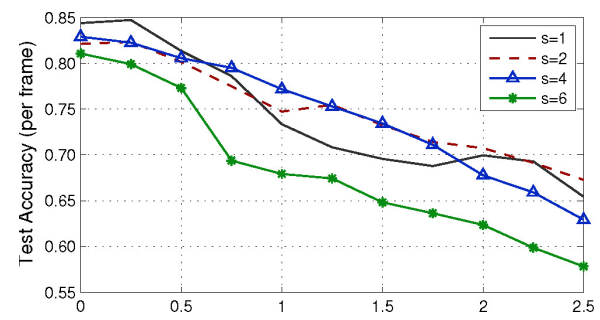

(d) BDH-5: Translation (units)

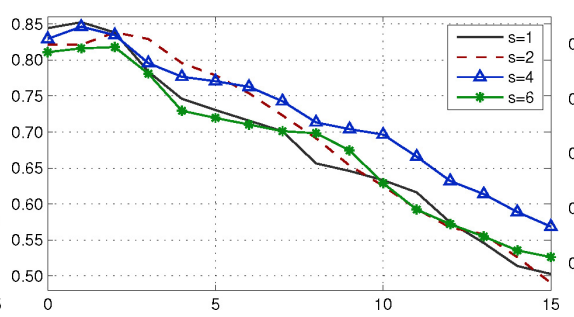

(e) BDH-5: Rotation (degrees)

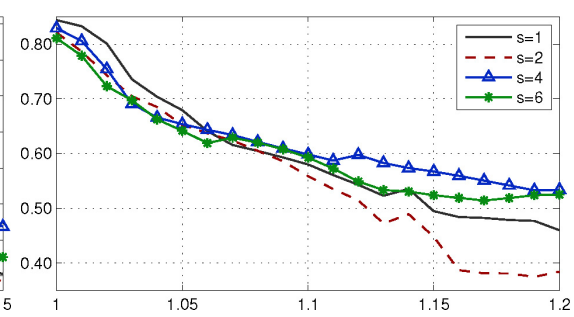

(f) BDH-5: Scaling (ratio)

Fig. 12 Invariance test results using only spatial thread on SD-5 (upper row) and BDH-5 (lower row) datasets; the test accuracy (averaged over 10 executions) is presented as a function of different transformations of the test frames. For translation (a, d), we moved each test frame by up to 4 (SD-5) and 2.5 (BDH-5) units in both directions horizontally and vertically using linear interpolation and zero padding, and calculated the averaged test accuracy over four moving directions. For rotation (b, e), we rotated each test frame by a degree of $-10^{\circ} \sim 10^{\circ}$ (SD-5) and $-15^{\circ} \sim 15^{\circ}$ (BDH-5) about the center point using bilinear interpolation and cropping strategies, and computed the averaged test accuracy over two rotation directions. For scaling (c, f), we amplified each testing frame by a ratio of up to 1.2 times using bicubic interpolation and cropping strategies.

datasets were carried out on Intel Core i7, 2.3-GHz CPU with 16-GB RAM.

\subsection{On Invariance Quality and OTConv Tile Size}

The theoretical proof of translational invariance for standard ConvNets with random filters and square pooling was given in [40]. As a standard ConvNet is unlikely to be scale and rotation invariant, authors of [36] found that tiling ConvNets are invariant to those transformations. Thus we carried out an empirical invariance analysis of OTConv based on prediction accuracy for tactile datasets of SD-5 and BDH-5 using only the individual tactile frames (ignore any temporal correlations among consecutive frames) as input. For the sake of result interpretability, we only use the minimal OTConv network defined in Fig. 2 (i.e. OTConv + square-root pooling) as feature extractor, the output of which forms a spatial feature space:

$$
\mathbf{P}_{\mathrm{s}}=\left[\begin{array}{c}
\mathbf{p}\left(\mathbf{x}^{(1)}\right) \\
: \\
\mathbf{p}\left(\mathbf{x}^{(N)}\right)
\end{array}\right]=\left[\begin{array}{ccc}
p_{1}\left(\mathbf{x}^{(1)}\right) & \cdots & p_{F}\left(\mathbf{x}^{(1)}\right) \\
: & : & : \\
p_{1}\left(\mathbf{x}^{(N)}\right) & \cdots & p_{F}\left(\mathbf{x}^{(N)}\right)
\end{array}\right] .
$$

Tactile frame $\mathbf{x}^{(t)}$ is fed to the minimal OTConv network with $F$ feature maps to generate a joint pooling activation $\mathbf{p}\left(\mathbf{x}^{(t)}\right)=\left[p_{1}\left(\mathbf{x}^{(t)}\right), \ldots, p_{F}\left(\mathbf{x}^{(t)}\right)\right]$, which is a row vector concatenating pooling outputs. $p_{i}\left(\mathbf{x}^{(t)}\right)$ is also a row vector with $(d-r+1)^{2}$ pooling activations for the $i$-th feature map. The unfolded row vectors of $\mathbf{P}_{\mathrm{s}}$ are fully connected (weighted by $\mathbf{W}_{\mathrm{s}}$ ) with $l$ output nodes that represent $l$ object classes. Finding the optimal values for $\mathbf{W}_{\mathrm{S}} \in \mathbb{R}^{\left[F \times(d-r+1)^{2}\right] \times l}=\left[\mathbf{w}_{1}, \ldots, \mathbf{w}_{F \times(d-r+1)^{2}}\right]^{\top}$ is a regularized least-squares problem, which is strictly convex, so its solution can be found by simply following [58]:

$$
\mathbf{W}_{\mathrm{s}}=\left\{\begin{array}{ll}
\mathbf{P}_{\mathrm{s}}^{\top}\left(\lambda \mathbf{I}+\mathbf{P}_{\mathrm{s}} \mathbf{P}_{\mathrm{s}}^{\top}\right)^{-1} \mathbf{Y}, \quad N \leq F(d-r+1)^{2} \\
\left(\lambda \mathbf{I}+\mathbf{P}_{\mathrm{s}}^{\top} \mathbf{P}_{\mathrm{s}}\right)^{-1} \mathbf{P}_{\mathrm{s}}^{\top} \mathbf{Y}, \quad N>F(d-r+1)^{2}
\end{array},\right.
$$

where $\mathbf{Y}$ is defined as $\left[\mathbf{y}^{(1)}, \ldots, \mathbf{y}^{(N)}\right]_{N \times l}^{\top} ; \mathbf{I}$ is the identity matrix of matching dimensionality; small positive scalar value $\lambda$ is added to improve result stability; when the training set becomes very large $(N \gg$ size of feature space), the second solution should be applied to reduce computational costs.

The key parameters [cf. (5) and Fig. 2] used to configure our invariance tests were $F=12, k=4, r=6$, and $C=10^{2}$. Because SD-5 and BDH-5 were collected with different tactile sensors (cf. Table 2) leading to different frame resolutions, we tested SD-5 with 6 tile sizes $s \in\{1,2,4,8,12,16\}$, but with 4 sizes $s \in\{1,2,4,6\}$ on BDH-5. At testing phase, three kinds of transformations were considered: translation (Fig. 12a, 12d), rotation (Fig. 12b, 12e), and scaling (Fig. 12c, 12f). We apply the corresponding transformation to all the test 
frames, and predict the label of the $t$-th frame with $\arg \max _{y \in\{1,2,3,4,5\}}\left[\mathbf{p}\left(\mathbf{x}^{(t)}\right) \cdot \mathbf{W}_{\mathrm{s}}\right]$.

From test results synthesized in Fig. 12, we discover when the transformation degree becomes increasingly large, the test accuracies kept dropping for all tile sizes. The only local exception happened in case of very slight changes (e.g. rotation $0^{\circ} \sim 1^{\circ}$ in Fig. 12e and scale $1 \sim$ 1.03 in Fig. 12c), where test accuracies turned out to be improved a little. This may be attributed to the interpolation algorithms applied to small transformations, which smoothened the adjacent "pixel" units within a single frame; this tiny growth of test accuracy could also be reproduced by simply smoothening test frames without any transformation. We also find that networks using reasonably larger tile sizes were apt to be more robust to the degree of translation, rotation, and scaling; but overly large tile size could overfit the model (e.g. the curve of $s=6$ in Fig. 12d). The comparison between the result patterns for SD-5 (Fig. 12a-12c) and BDH5 (Fig. 12d-12f) suggests that partially untied weights seems producing better invariance quality for higherresolution tactile sensors than that containing fewer sensor units, which practically promotes the necessity to install high-resolution sensors on industrial robots.

\subsection{Optimal Stack-Size of Network Input}

The intention of this section is to find out the optimal input stack-size. The stack-size is noted as $n$ (cf. Table 1 and section 4.1), which indicates using $n+1$ individual frames and $n$ tactile-flow slices as dual input of ROTConvPCE. Intuitively, using a larger $n$ enables capturing temporal dynamics over longer time span, but also increases the amount of learnable network parameters dramatically, making the training a lot more time-consuming. As a consequence, it becomes important to find an optimal $n$ that can reach a good balance between recognition performance and the network scale. For this purpose, we test the performance of ROTConvPCE (without majority voting) towards all seven tactile tasks using 15 different stack-sizes: $n \in$ $\{1,2, \ldots, 15\}$. The test accuracies shown in Fig. 13 were averaged over five trainings, each of which used a 7:3 train/test-ing split.

The observations from Fig. 13 are two fold: (1) the test accuracies almost monotonically improve until the stack-size reaches approximately 10, while 3T-RTCN [33] stopped improving at $n=2$, implying the new architecture can extract more global temporal information from deeper frame stacks; (2) unlike 3T-RTCN, ROTConvPCE does not explicitly use intensity difference information, but it obtains better accuracies than 3TRTCN on all datasets, which may attribute to the new

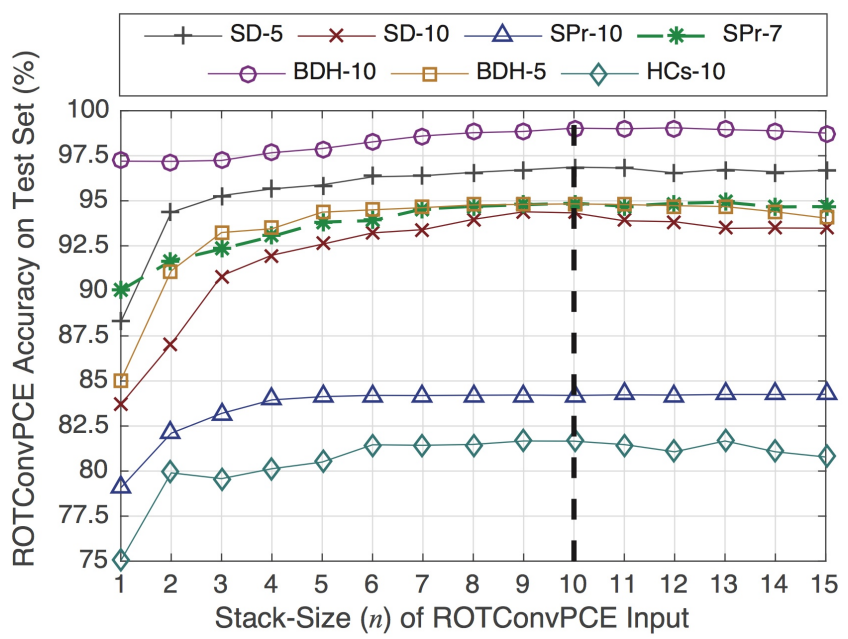

Fig. 13 ROTConvPCE inference accuracy on test dataset (test accuracy) as a function of the input stack-size.

architecture's ability in extracting much richer temporal features (implicitly including intensity differences) from stacked frames. It is noteworthy that increasing the stack-size from 10 to larger values only leads to a trivial enhancement or even worse performance, so we use $n=10$ for the experiments that followed.

\subsection{Comparison with Randomized Networks}

In contrast to 3T-RTCN, ROTConvPCE has a deeper structure that requires weight-finetune iterations, hence it inevitably needs significantly longer training time to learn a model with good generalization ability. However, the activity of model training rarely demands constant execution: because when the model is properly trained, it will be deployed as an inference service in real applications to carry out predictions using input data. Observed from Fig. 14a that compares the framelevel prediction accuracies between ROTConvPCE and 3T-RTCN, we see that ROTConvPCE outperformed $3 \mathrm{~T}$-RTCN with an average gap of $3.5 \%$, which makes us curious about the answer to this question: is this improvement a result of sacrificing inference efficiency?

As a randomized approach, 3T-RTCN has already been reported as a fast algorithm during both training and testing phase [33]; therefore we mainly use 3TRTCN as a benchmark method to answer the aforementioned question. We deploy the trained model of 3T-RTCN (Matlab) and ROTConvPCE (Caffe) on the same physical server, and send batches (batch size: 128) of testing samples for synchronized prediction; then we calculate the average time spent in predicting one single sample. Fig. 14b shows that despite of the overhead of finetuning and deeper network structure, ROTCon- 
Table 3 Performance comparison of ROTConvPCE-mv and state-of-the-art methods: "acc.": average testing accuracies (\%) and "dev.": standard deviations of testing accuracies.

\begin{tabular}{|c|c|c|c|c|c|c|c|c|c|c|c|c|c|c|c|}
\hline \multirow{2}{*}{$\begin{array}{c}\text { Dataset } \\
\text { [Ref.] }\end{array}$} & \multicolumn{2}{|c|}{$\begin{array}{c}\text { ROTConvPCE } \\
\text {-mv (Ours) }\end{array}$} & \multicolumn{2}{|c|}{$\begin{array}{c}\text { 3T-RTCN } \\
{[33]}\end{array}$} & \multicolumn{2}{|c|}{$\begin{array}{c}\text { JKSC } \\
{[23]}\end{array}$} & \multicolumn{2}{|c|}{$\begin{array}{c}\text { MV-HMP } \\
{[6,59]}\end{array}$} & \multicolumn{2}{|c|}{$\begin{array}{c}\text { ST-HMP } \\
{[6]}\end{array}$} & \multicolumn{2}{|c|}{$\begin{array}{c}\text { LDS-Martin } \\
{[60,26]}\end{array}$} & \multicolumn{2}{|c|}{$\begin{array}{c}\text { BoS-LDSs } \\
{[5]}\end{array}$} & \multirow[t]{2}{*}{ Other Models } \\
\hline & acc. & dev. & acc. & dev. & acc. & dev. & acc. & dev. & acc. & dev. & acc. & dev. & acc. & dev. & \\
\hline SD-5 $[19,11]$ & 100 & 0.0 & 100 & 0.0 & 92.0 & 1.35 & $90.5^{*}$ & $1.80^{*}$ & $\underline{98.9}$ & $2.99^{*}$ & 97.0 & 1.60 & 96.8 & 1.12 & $91.6^{*}\left(\mathrm{HMM}_{\mathrm{LR}}\right)[19,11]$ \\
\hline $\mathrm{SD}-10[19,11]$ & 100 & 0.0 & 100 & 0.0 & 91.5 & 2.87 & $94.0^{*}$ & $7.00^{*}$ & $94.0^{*}$ & $7.00^{*}$ & 92.0 & 3.02 & ${\underline{97.5^{*}}}^{*}$ & - & $97.0^{*}(\mathrm{MV}-\mathrm{HMP} \mathrm{FD})[6]$ \\
\hline SPr-10 [22] & 100 & 0.0 & 100 & 0.0 & $87.0^{*}$ & - & $84.5^{*}$ & $18.2^{*}$ & $88.5^{*}$ & $12.9^{*}$ & $\underline{94.5}$ & 6.45 & $\overline{94.2}$ & 5.30 & $91.1^{*}\left(\mathrm{ST}-\mathrm{HMP} \mathrm{P}_{\mathrm{FD}}\right)[6]$ \\
\hline SPr-7 [22] & 100 & 0.0 & 100 & 0.0 & $91.4^{*}$ & - & $94.3^{*}$ & $7.40^{*}$ & $95.7^{*}$ & $6.90^{*}$ & 97.1 & 3.98 & $\underline{98.6}$ & - & $97.1^{*}\left(\mathrm{ST}-\mathrm{HMP} \mathrm{FD}_{\mathrm{F}}\right)[6]$ \\
\hline BDH-10 [4] & 100 & 0.0 & 100 & 0.0 & $\underline{94.0}$ & 3.20 & 81.6 & 6.50 & 87.5 & 5.94 & 82.0 & 4.06 & 90.5 & 2.71 & $96.0^{*}(\mathrm{pLDSs})[4]$ \\
\hline BDH-5 [23] & 100 & 0.0 & 100 & 0.0 & $87.0^{*}$ & - & 76.8 & 8.82 & 81.0 & 7.71 & $\underline{98.0}$ & 3.60 & 94.6 & 2.69 & $\mathbf{1 0 0 . 0}^{*}(\mathrm{LDS}-\mathrm{SC})[26]$ \\
\hline HCs-10 $[14,33]$ & 97.5 & 0.37 & 91.2 & 2.02 & $\underline{93.5}$ & 1.48 & 67.7 & 9.40 & 83.0 & 6.95 & 70.0 & 4.55 & 74.5 & 4.15 & - \\
\hline
\end{tabular}

${ }^{*}$ An asterisk designates the best reported testing score directly referenced from the corresponding paper(s); and therefore the corresponding standard deviation is mostly unavailable.

Table 4 The comparison of the number of free parameters to be learned in different state-of-the-art models.

\begin{tabular}{|c|c|c|c|c|c|}
\hline Dataset [Ref.] & ROTConvPCE-mv (Ours) & 3T-RTCN [33] & $\mathrm{JKSC}^{*}[23]$ & $\mathrm{MV}-\mathrm{HMP}^{\dagger} \mathrm{ST}-\mathrm{HMP}^{\dagger}[6,59]$ & $\operatorname{LDS}-\operatorname{Martin}^{\ddagger}[60,26]$, BoS-LDSs ${ }^{\ddagger}[5]$ \\
\hline $\mathrm{SD}-5[19,11]$ & 567,168 & 546,764 & 16,986 & 97,200 & 228,160 \\
\hline $\mathrm{SD}-10[19,11]$ & 572,288 & 809,688 & 32,700 & 46,800 & 219,600 \\
\hline $\mathrm{SPr}-10[22]$ & 572,288 & 536,282 & 16,878 & 25,600 & 120,060 \\
\hline SPr-7 $[22]$ & 569,216 & 167,118 & 8,820 & 25,600 & 86,940 \\
\hline $\mathrm{BDH}-10[4]$ & 572,288 & 153,364 & 8,256 & 20,800 & 53,580 \\
\hline $\mathrm{BDH}-5[23]$ & 564,096 & 14,128 & 27,000 & 14,400 & 73,800 \\
\hline HCs-10 $[14,33]$ & 572,288 & 5,866 & 29,160 & 3,200 & 42,120 \\
\hline
\end{tabular}

* We treat one computed DTW kernel as one free parameter tuned; and the dictionary size is equal to the size of training dataset.

$\dagger$ The number of code words is set to 200 in these cases; and the parameters of the final SVM classifier are not counted.

$\ddagger$ The dimension of the LDS hidden state vector is set to 10 for fair comparison in the above table; and the number of parameters in the classifier is excluded.

vPCE mostly and consistently consumed much less time during inferencing, which may be a consequence of either factors: (1) less input threads and fully-connected layers, and (2) highly efficient implementation of batch feed-forward computation in Caffe. Moreover, due to the interpolation step, ROTConvPCE shows another superior characteristic: the inference time becomes irrelevant to the dimension of input frames, which is favored by many applications.

\subsection{On Comparison with the State-of-the-Arts}

This section intends to compare ROTConvPCE-mv with several state-of-the-art models designed for tactile recognition tasks; they are 3T-RTCN [33], JKSC ${ }^{2}$ [23], MVHMP (Majority-Voting HMP), ST-HMP 3 [59,6], LDSMartin (LDS based on Martin-distance) ${ }^{4}[60,26]$, and BoS-LDSs (LDSs in Bag-of-Systems) ${ }^{5}$ [5]. Besides, we also refer to other models that had reported competitive results on a few tactile datasets; these models are $\mathrm{ST} / \mathrm{MV}-\mathrm{HMP}_{\mathrm{FD}}$ (i.e. ST/MV-HMP with a 3D dic-

\footnotetext{
2 Building on the JKSC source code provided by the authors of [23], we parallelized the joint-kernel computation and replaced the code for DTW (Dynamic Time Wrapping) calculation with a compiled C binary: www.mathworks.com/ matlabcentral/profile/authors/2797017-quan-wang.

3 MV/ST-HMP: https://github.com/mmadry/st-hmp

${ }^{4}$ http://vision.ucla.edu/ doretto/projects/dynamicrecognition.html

5 Test scores for BoS-LDSs are provided by authors of [5].
}

tionary) [6], $\mathrm{HMM}_{\mathrm{LR}}$ (Left-Right HMM) [19,11], and pLDSs (piecewise LDSs) [4]. For fair comparability with some directly referenced results (i.e. the asterisked scores in Table 3), all simulations were carried out on 10-fold cross-validations using 9:1 splits. The highest score for each dataset is indicated in bold; and the second best one is underlined.

\subsubsection{The Average Testing Accuracy}

It can be seen from Table 3 that our network achieved the best recognition accuracy on all tasks. It is emphasized in [33] that 3T-RTCN is suboptimal than JKSC for modeling low-dimensional tactile data (e.g. HCs10), because convolution and pooling will cause a big portion of granularity loss, which may undermine the recognition rate. Nevertheless, ROTConvPCE-mv prevents that problem by introducing interpolation, zero-padding OTConvs, and a hierarchical OTConv ensemble; thus it managed to achieve a much higher accuracy (97.5\%) than both 3T-RTCN and JKSC. Additionally, the standard deviation of testing accuracies can largely rank the compared methods by their relative performance variance (e.g. from high to low): MV-HMP $\geq$ ST-HMP $\geq$ LDSMartin $\geq$ BoS-LDS $\geq$ JKSC $\geq 3$ T-RTCN $\geq$ ROTConvPCEmv. 


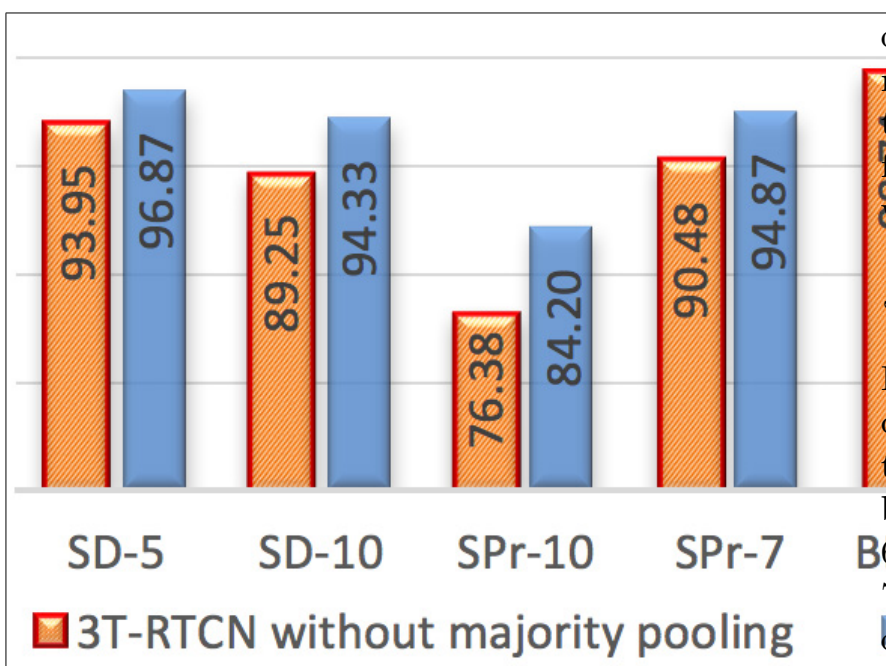

overly small number of parameters (e.g.1 $106 s-10)$. Our network uses constantly a large amotint of parameters to 1) menable learning over-complete and hierarchical fea uros, and 2 prenenting to a large extêt the tedious oo k of hyper rameter tuning.

6)

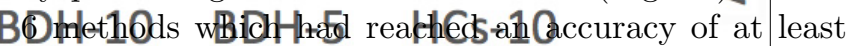
$70 \%$. Seen from Fig. 15e and 15f, LDS-Martin lost out

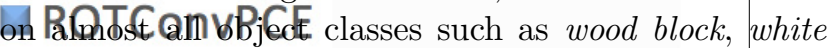
(a) Average prediction/test acce, and metglgl lock particularly; and BoS-LDSs greatly improved its recognition rate for the first two categories.

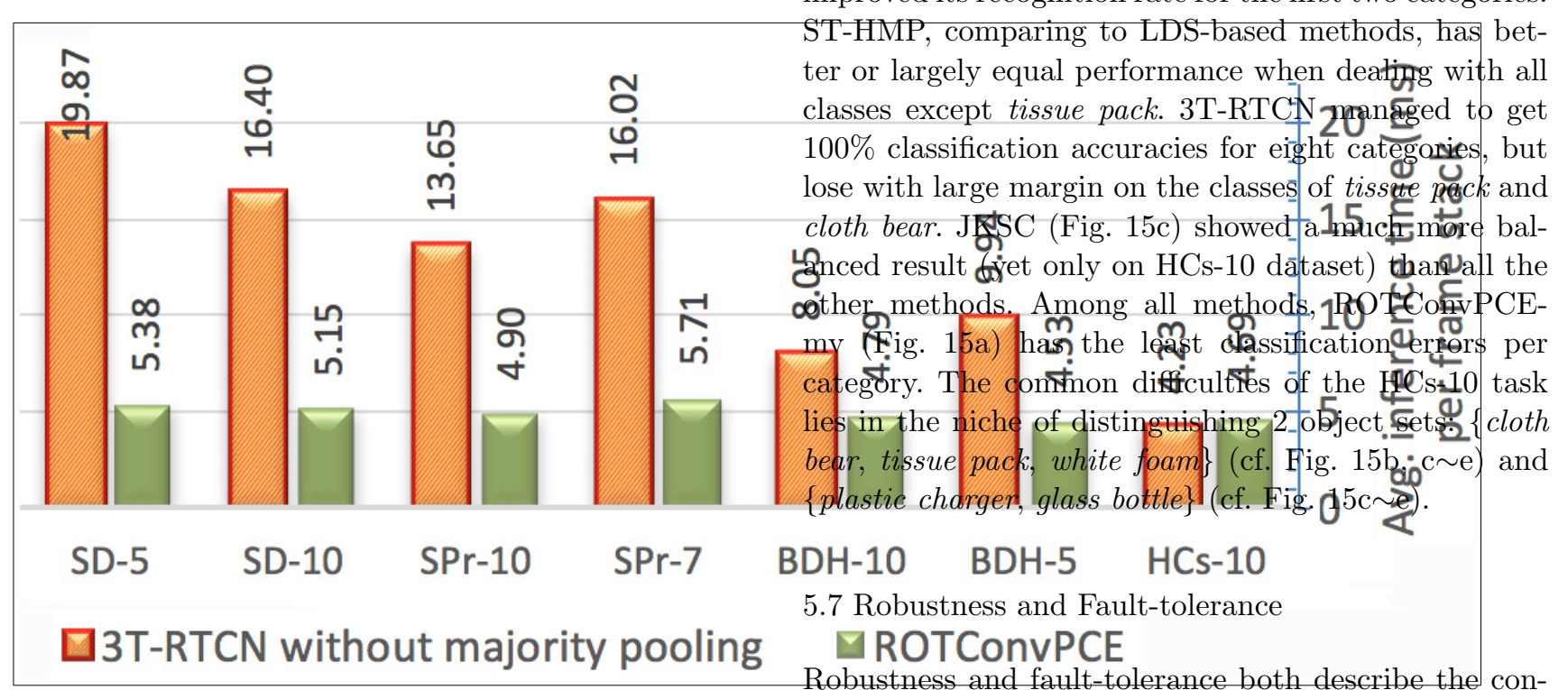

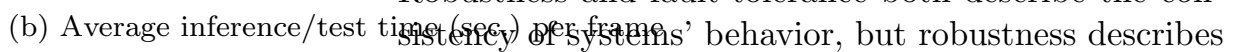

Fig. 14 The comparison of (a) prediction accuracy and (b) inference time between ROTConvPCE and 3T-RTCN.

\subsubsection{The Amount of Learnable Parameters}

The size of different models is also compared (Table 4) in terms of the number of free parameters to be trained. Although models of JKSC and MV/ST-HMP have the smallest sizes, we also empirically notice that they constantly trained much slower than our network, which may be the consequence of the time-consuming activities of kernel computation and dictionary learning. LDS based algorithms (e.g. LDS-Martin/BoS-LDSs) mostly achieved the fastest training speed using a mediumsized model. The model size of 3T-RTCN is tightly coupled with both the input-size and the hyper-parameters, which may result in under-fitting because of using an the response to the input, while fault-tolerance describes the response to the dependent environment. Johnson et al. [61] formally defined a fault-tolerant system as the one that can continue its intended task (possibly at a reduced level) rather than failing completely, if the system partially fails. We will compare ROTConvPCEmv with four models: 3T-RTCN, JKSC, ST-HMP, and LDS-Martin, which represent four mainstream methodologies: i.e. neural networks, sparse coding, unsupervised feature learning, and time-series modeling.

\subsubsection{Robustness to Sensor Noise}

We manually added different capacities of noise (white Gaussian) to frame vectors. SNR (Signal-to-Noise Ratio) is used as a measure for comparing the strength of the desired force signal to the level of background noise. It is usually defined as the ratio of signal strength 


\begin{tabular}{cccccccccccc}
\hline & 1 & 2 & 3 & 4 & 5 & 6 & 7 & 8 & 9 & 10 \\
1 & 100 & 0 & 0 & 0 & 0 & 0 & 0 & 0 & 0 & 0 \\
2 & 0 & 100 & 0 & 0 & 0 & 0 & 0 & 0 & 0 & 0 \\
3 & 0 & 0 & 100 & 0 & 0 & 0 & 0 & 0 & 0 & 0 \\
4 & 0 & 0 & 0 & 100 & 0 & 0 & 0 & 0 & 0 & 0 \\
5 & -10 & 0 & 0 & 0 & 90 & 0 & 0 & 0 & 0 & 0 \\
6 & 0 & 0 & 0 & 0 & 0 & 100 & 0 & 5 & 0 & 0 \\
7 & 0 & 0 & 0 & 0 & 5 & 0 & 85 & 0 & 0 & 5 \\
8 & 0 & 0 & 0 & 0 & 0 & 0 & 0 & 100 & 0 & 0 \\
9 & 0 & 0 & 0 & 0 & 0 & 0 & 0 & 0 & 100 & 0 \\
10 & 0 & 0 & 0 & 0 & 0 & 0 & 0 & 0 & 0 & 100
\end{tabular}

(a) ROTConvPCE-mv

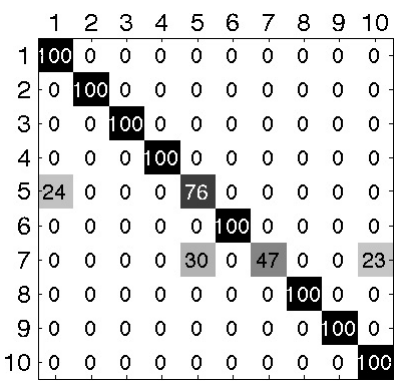

(b) $3 \mathrm{~T}-\mathrm{RTCN}$

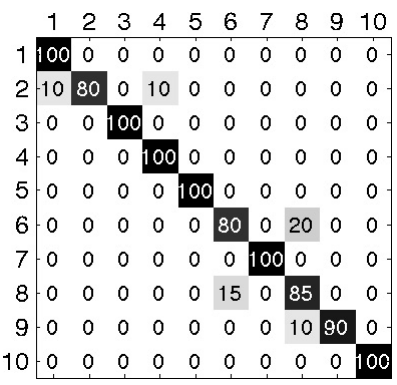

(c) JKSC

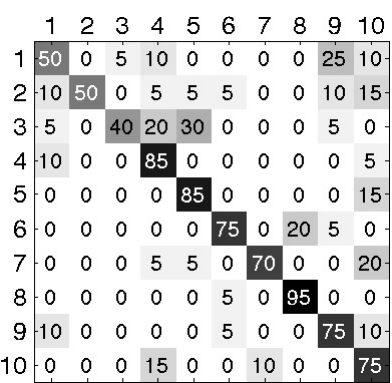

(e) LDS-Martin

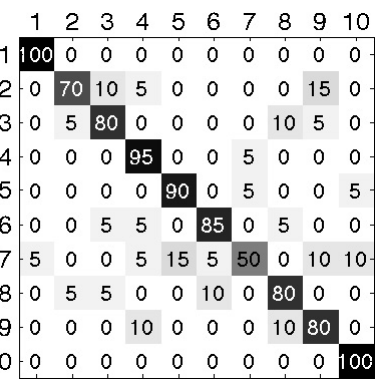

(d) ST-HMP

$\begin{array}{llllllllll}1 & 2 & 3 & 4 & 5 & 6 & 7 & 8 & 9 & 10\end{array}$

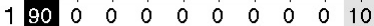
$200 \begin{array}{llllllllll} & 80 & 0 & 5 & 5 & 5 & 0 & 0 & 5 & 0\end{array}$

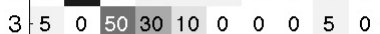
$\begin{array}{lllllllllll}4 & 5 & 0 & 0 & 70 & 0 & 0 & 0 & 0 & 10 & 15\end{array}$ $5-0 \begin{array}{llllllllll}0 & 0 & 0 & 0 & 85 & 0 & 0 & 0 & 0 & 15\end{array}$ $6 \cdot \begin{array}{lllllllllll}0 & 0 & 0 & 0 & 0 & 75 & 0 & 25 & 0 & 0\end{array}$ $\begin{array}{cccccccccccc}7 & -10 & 0 & 0 & 0 & 5 & 0 & 60 & 0 & 0 & 25 \\ 8 & 0 & 5 & 0 & 5 & 0 & 10 & 0 & 80 & 0 & 0\end{array}$ $8 \begin{array}{lllllllllll}8 & 5 & 0 & 5 & 0 & 10 & 0 & 80 & 0 & 0\end{array}$

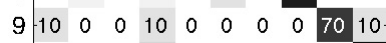
$10-0 \begin{array}{llllllllll}0 & 0 & 0 & 15 & 0 & 0 & 0 & 0 & 0 & 85\end{array}$

(f) BoS-LDSs
Fig. 15 The confusion matrices of 6 methods reaching a minimum test accuracy of $70 \%$ on HCs- 10 dataset. The object classes: 1-wood block, 2-white tape, 3-metal lock, 4-rubber stamp, 5-cloth bear, 6-plastic charger, 7-tissue pack, 8-glass bottle, 9-staple box, 10-white foam. to noise power, and often expressed in decibels (dB): $\mathrm{SNR}_{\mathrm{dB}}=10 \cdot \log _{10}\left(P_{\mathrm{sig}} / P_{\mathrm{nos}}\right)$, where $P_{\mathrm{sig}}$ and $P_{\text {nos }}$ denote the power of signal and noise respectively; and $\mathrm{SNR}>0$ indicates larger portion of signal than noise. Figure 16 demonstrates that ROTConvPCE-mv offered the best noise robustness quality. 3T-RTCN still maintained strong classification capability even when the noise strength ratio reached up to $50 \%(\mathrm{SNR}=0)$; but with the same amount of noise contamination, other models' performance dropped dramatically without exception. ST-HMP turned out to have the best noise robustness among the rest of the referenced methods, while JKSC had relatively the worst one that sometimes lost its classification power completely at $\mathrm{SNR}=0$.

\subsubsection{Fault-tolerance to Partial Sensor Malfunction}

A common hardware glitch of tactile sensors is partial sensor malfunction, in which one or more units composing the sensor array are broken and hence always output a fixed value (most likely being zero) or random values; and it is prone to occur in extreme environments such as fire scene (with high temperature and deleterious gas), frozen lake (with low temperature and humid conditions), and outer space (with high ultra-violet index, exceedingly thin air, and dramatic temperature change). To examine the fault-tolerance ability in coping with such malfunction, we intentionally sabotaged a percentage $(1 \sim 100 \%$ with rounding scheme) of unit readings. We carried out two groups of simulations: one group (top row in Figure 17) used zeros to replace the "damaged" units; the other group (bottom row) made them output random values from the range of $[0,1]$. For each malfunction ratio, five sets of "broken" units were picked, so that each data point is in-fact the average test accuracy of $10 \times 5=50$ simulations. Figure 17 evidently validates that both ROTConvPCE-mv and 3T-RTCN provided much more superior fault-tolerance ability (to sensor malfunction) than other methods; and ROTConvPCE-mv managed to achieve even more robust performance than 3T-RTCN in most occasions. Another instructive and illuminating discovery is that all models (esp. JKSC and LDS-Martin) cope with the zero signal better than the noise "generated" by malfunction units; it hence implies detecting malfunction units and forcing them to output zero values might be a beneficial approach for tactile recognition.

\subsubsection{Fault-tolerance to Frame Loss}

Another repeatedly seen corruption of the tactile sensory data is frame loss, which is usually caused by transmission circuit malfunction. Frame loss occurs when 


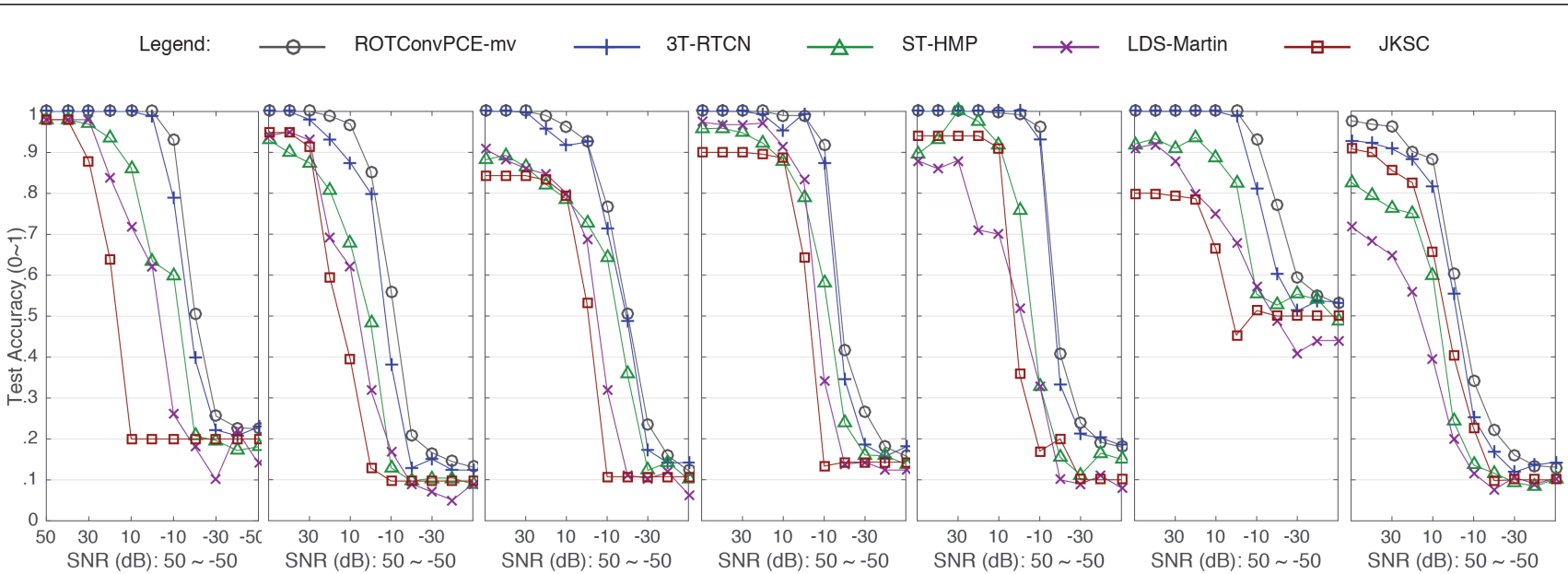
(a) SD-5
(b) $\mathrm{SD}-10$
(c) $\mathrm{SPr}-10$
(d) $\mathrm{SPr}-7$
(e) BDH-10
(f) BDH-5
(g) HCs-10

Fig. 16 Performance of robustness: testing accuracy as a function of simulated sensor noise measured by SNR.

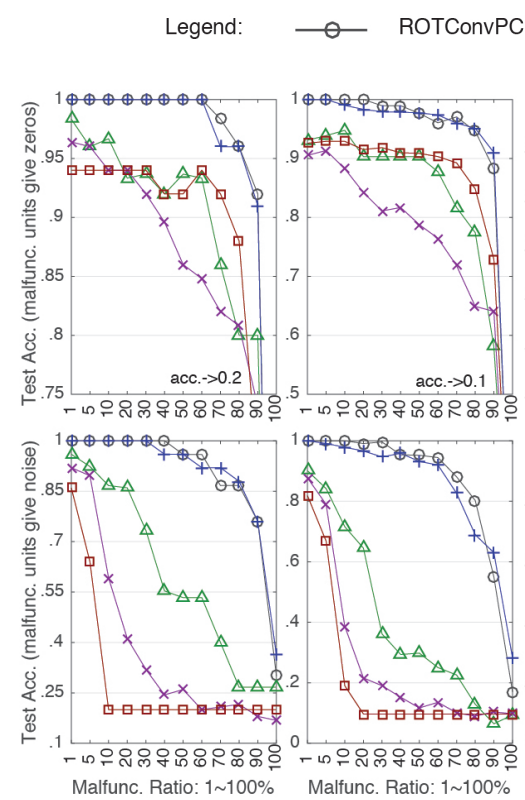

(a) SD-5

(b) SD-10

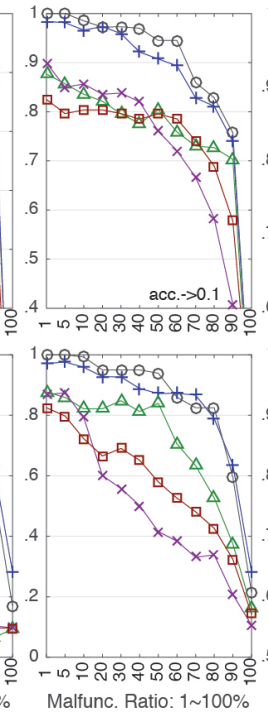

(c) $\mathrm{SPr}-10$
3T-RTCN

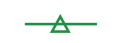

ST-HMP

$\longrightarrow$ LDS-Martin

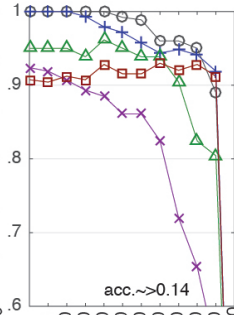

-
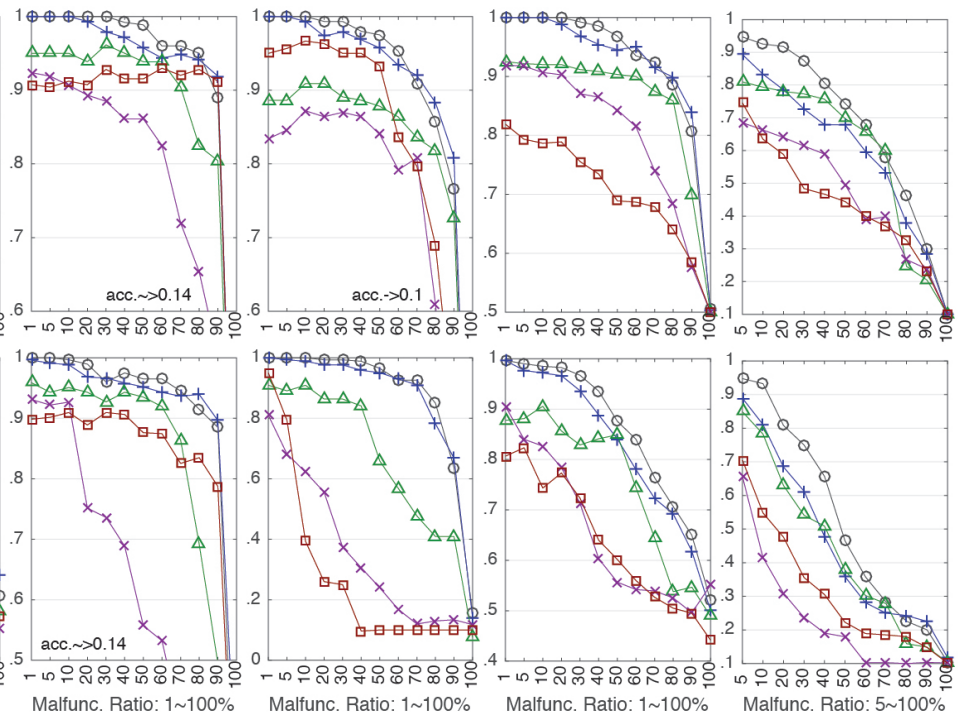

(d) $\mathrm{SPr}-7$

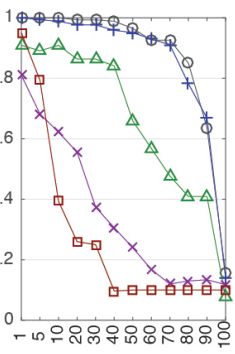

(e) BDH-10

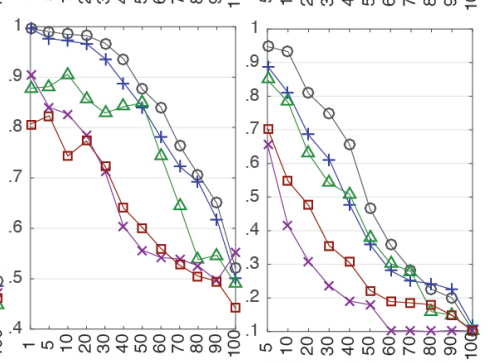

(f) BDH-5

(g) HCs-10

Fig. 17 Fault-tolerance capability to partial sensor malfunction: average testing accuracy as a function of malfunction ratio. Experimental results for two simulation groups are presented: top row - the malfunction units output constant zero values; bottom row - the malfunction units output random noise values.

some frame slices sent across the transmission circuit fail to reach their destinations of storage. As another kind of hardware malfunction, just like partial sensor malfunction, it is also inclined to happen in many enormously hazardous environments. To simulate frame-loss context, we arbitrarily removed a ratio of frames from each sequence; and for every frame-loss ratio, we made five random choices of throwaway frames. From Figure 18, we found that algorithms are more tolerable to frame-loss than sensor malfunction, because frame-loss primarily ruins the temporal coherence between neighboring frames while keeping the spatial information in- tact. The performance of ROTConvPCE-mv and 3TRTCN slowly and monotonically decreased upon higher frame-loss ratio; nevertheless they performed constantly better than other models at almost any presumed frameloss ratio. With the newly proposed network architecture, ROTConvPCE-mv generally obtained an even better frame-loss tolerance performance than 3T-RTCN. We also notice that ST-HMP was hardly impacted by frame-loss; hence in this sense, ST-HMP is a stable tactile feature learning algorithm. Interestingly, the frameloss tolerance trend of JKSC varied dramatically on different datasets; its test accuracy could even conspic- 

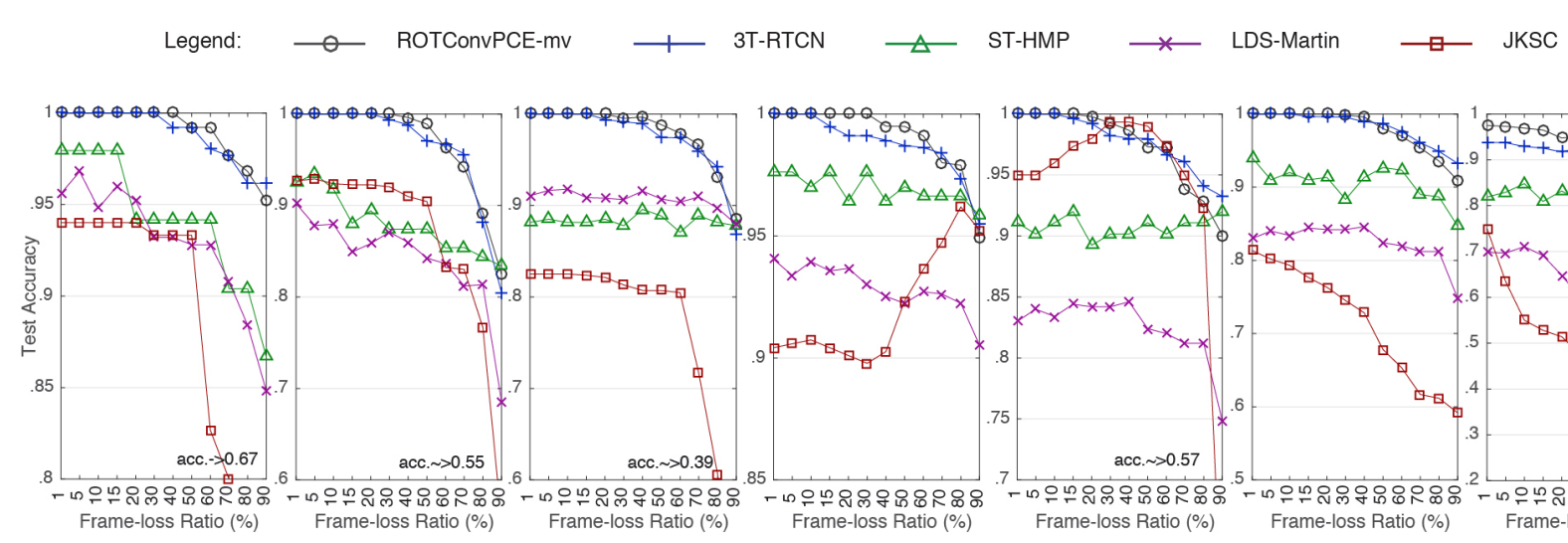

(a) SD-5

(b) SD-10

(c) $\mathrm{SPr}-10$

(d) $\operatorname{SPr}-7$
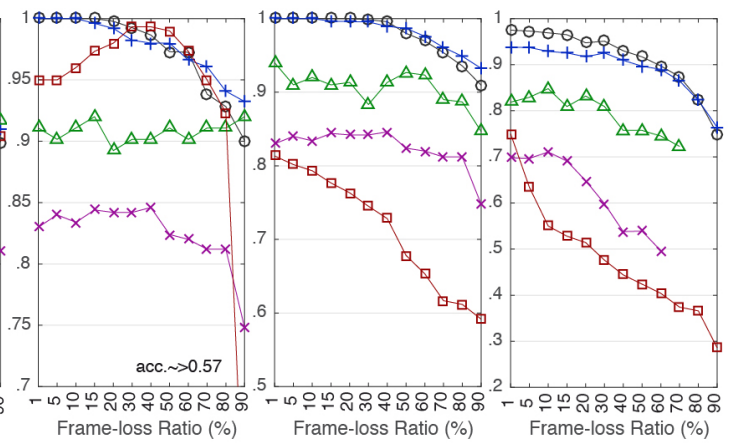

(e) $\mathrm{BDH}-10$

(f) $\mathrm{BDH}-5$

(g) HCs-10

Fig. 18 Fault-tolerance to frame loss caused by transmission circuit malfunction: mean acc. as a function of frame-loss ratio.

uously go up with more lost frames (Fig. 18d,e); our assumption is that frame-loss works like subsampling that eliminates the local peak-and-trough of tactile output along the time axis; but it is hard to take any advantage of this phenomenon, since over-subsampling can also do great harm to the performance.

\section{Conclusions and Perspectives}

In this paper, we put forward the new OTConv layers followed by the introduction of ROTConv modules and the PCE ensemble, which altogether constitute our ROTConvPCE-mv model; this end-to-end architecture performs effective tactile recognition using stacks of individual frames and tactile flows as input. We empirically discovered that the ROTConvs using reasonably larger tile sizes turned out to be more robust to the transformations of translation, rotation, and scaling; but overly large tile size could overfit the model. ROTConvPCE-mv outperformed several state-of-theart methods by a large margin on inferencing efficiency, prediction accuracy, robustness, and fault-tolerance capability. In comparison with our earlier model 3T-RTCN the new model is capable of capturing global temporal correlations from longer time span, and also generally superior (or at least equivalent) in almost all of our experimental evaluations. We also noted that forcing the malfunction units generating zero values is potentially beneficial in enhancing the fault-tolerance ability. Our future perspectives include addressing larger-scaled tactile data, investigating the possibility of tailoring the recurrent neural network for efficient tactile recognition, and try to explain at the convergence the features extracted by the convolution layers.

\section{Compliance with Ethical Standards}

Funding This work was supported by grants from the China National Natural Science Foundation (No. 61327809 and 61210013). Lele Cao is also supported by the State Scholarship Fund under file number 201406210275.

Conflict of Interest Lele Cao, Fuchun Sun, Xiaolong Liu, Wenbing Huang, Ramamohanarao Kotagiri, and Hongbo Li declare that they have no conflict of interest. Human and Animal Rights This article does not contain any studies with human or animal subjects performed by any of the authors.

Acknowledgements We thank Weihao Cheng for suggesting momentum prediction [62] during the process of iterating our work. We also express our appreciation to Jingwei Yang and Rui Ma for the explanation of JKSC and BoS-LDSs source code. We also express our gratitude to Xiaohui $\mathrm{Hu}$ and Haolin Yang for their help in collecting the HCs10 dataset.

\section{References}

1. Sun, F., Liu, C., Huang, W., Zhang, J.: Object classification and grasp planning using visual and tactile sensing. IEEE Transactions on Systems Man and Cybernetics: Systems 46(7), 969-979 (2016)

2. Kappassov, Z., Corrales, J.A., Perdereau, V.: Tactile sensing in dexterous robot hands. Robotics and $\mathrm{Au}-$ tonomous Systems 74, 195-220 (2015)

3. Xu, D., Loeb, G.E., Fishel, J.A.: Tactile identification of objects using bayesian exploration. In: Proc. of ICRA, pp. 3056-3061 (2013)

4. Xiao, W., Sun, F., Liu, H., He, C.: Dexterous robotic hand grasp learning using piecewise linear dynamic systems model. In: Proc. of ICCSIP, pp. 845-855 (2014)

5. Ma, R., Liu, H., Sun, F., Yang, Q., Gao, M.: Linear dynamic system method for tactile object classification. Science China Information Sciences 57(12), 1-11 (2014)

6. Madry, M., Bo, L., Kragic, D., Fox, D.: ST-HMP: Unsupervised spatio-temporal feature learning for tactile data. In: Proc. of ICRA, pp. 2262-2269 (2014) 
7. Spiers, A.J., Liarokapis, M.V., Calli, B., Dollar, A.M.: Single-grasp object classification and feature extraction with simple robot hands and tactile sensors. IEEE transactions on haptics 9(2), 207-220 (2016)

8. Liu, H., Greco, J., Song, X., Bimbo, J., Seneviratne, L., Althoefer, K.: Tactile image based contact shape recognition using neural network. In: Proc. of MFI, pp. 138-143 (2012)

9. Hoelscher, J., Peters, J., Hermans, T.: Evaluation of tactile feature extraction for interactive object recognition. In: Proc. of IEEE-RAS 15th International Conference on Humanoid Robots (Humanoids), pp. 310-317. IEEE (2015)

10. Matsubara, T., Shibata, K.: Active tactile exploration with uncertainty and travel cost for fast shape estimation of unknown objects. Robotics and Autonomous Systems 91, 314-326 (2017)

11. Bekiroglu, Y., Laaksonen, J., Jorgensen, J.A., Kyrki, V., Kragic, D.: Assessing grasp stability based on learning and haptic data. IEEE Transactions on Robotics 27(3), 616-629 (2011)

12. Dang, H., Allen, P.K.: Stable grasping under pose uncertainty using tactile feedback. Autonomous Robots 36(4), 309-330 (2014)

13. Kwiatkowski, J., Cockburn, D., Duchaine, V.: Grasp stability assessment through the fusion of proprioception and tactile signals using convolutional neural networks. In: Proc. of IROS, pp. 286-292. IEEE (2017)

14. Yang, H., Liu, X., Cao, L., Sun, F.: A new slip-detection method based on pairwise high frequency components of capacitive sensor signals. In: Proc. of ICIST, pp. 56-61 (2015)

15. Heyneman, B., Cutkosky, M.R.: Slip classification for dynamic tactile array sensors. The International Journal of Robotics Research 35(4), 404-421 (2016)

16. Gorges, N., Navarro, S.E., Goger, D., Worn, H.: Haptic object recognition using passive joints and haptic key features. In: Proc. of of ICRA, pp. 2349-2355 (2010)

17. Luo, S., Mou, W., Althoefer, K., Liu, H.: Novel tactilesift descriptor for object shape recognition. IEEE Sensors Journal 15(9), 5001-5009 (2015)

18. Corradi, T., Hall, P., Iravani, P.: Bayesian tactile object recognition: Learning and recognising objects using a new inexpensive tactile sensor. In: Proc. of ICRA, pp. 3909$3914(2015)$

19. Bekiroglu, Y., Kragic, D., Kyrki, V.: Learning grasp stability based on tactile data and HMMs. In: Proc. of RO-MAN, pp. 132-137 (2010)

20. Soh, H., Su, Y., Demiris, Y.: Online spatio-temporal gaussian process experts with application to tactile classification. In: Proc. of IROS, pp. 4489-4496 (2012)

21. Gogulski, J., Boldt, R., Savolainen, P., Guzmán-López, J., Carlson, S., Pertovaara, A.: A segregated neural pathway for prefrontal top-down control of tactile discrimination. Cerebral Cortex (New York, NY: 1991) 25(1), 161-166 (2013)

22. Drimus, A., Kootstra, G., Bilberg, A., Kragic, D.: Design of a flexible tactile sensor for classification of rigid and deformable objects. Robotics and Autonomous Systems 62(1), 3-15 (2014)

23. Liu, H., Guo, D., Sun, F.: Object recognition using tactile measurements: Kernel sparse coding methods. IEEE Transactions on Instrumentation and Measurement 65(3), 656-665 (2016)

24. Chebotar, Y., Hausman, K., Su, Z., Sukhatme, G.S., Schaal, S.: Self-supervised regrasping using spatiotemporal tactile features and reinforcement learning. In: Proc. of IROS, pp. 1960-1966 (2016)
25. Wu, H., Jiang, D., Gao, H.: Tactile motion recognition with convolutional neural networks. In: Proc. of IROS, pp. 1572-1577 (2017)

26. Huang, W., Sun, F., Cao, L., Zhao, D., Liu, H., Harandi, M.: Sparse coding and dictionary learning with linear dynamical systems. In: Proc. of CVPR, pp. 3938-3947 (2016)

27. Karpathy, A., Toderici, G., Shetty, S., Leung, T., Sukthankar, R., Fei-Fei, L.: Large-scale video classification with convolutional neural networks. In: Proc. of CVPR, pp. 1725-1732 (2014)

28. Tu, Z., Zheng, A., Yang, E., Luo, B., Hussain, A.: A biologically inspired vision-based approach for detecting multiple moving objects in complex outdoor scenes. Cognitive Computation 7(5), 539-551 (2015)

29. Tu, Z., Abel, A., Zhang, L., Luo, B., Hussain, A.: A new spatio-temporal saliency-based video object segmentation. Cognitive Computation 8(4), 629-647 (2016)

30. Tünnermann, J., Mertsching, B.: Region-based artificial visual attention in space and time. Cognitive Computation 6(1), 125-143 (2014)

31. Simonyan, K., Zisserman, A.: Two-stream convolutional networks for action recognition in videos. In: Proc. of NIPS, pp. 568-576 (2014)

32. Guo, D., Sun, F., Fang, B., Yang, C., Xi, N.: Robotic grasping using visual and tactile sensing. Information Sciences 417, 274-286 (2017)

33. Cao, L., Kotagiri, R., Sun, F., Li, H., Huang, W., Aye, Z.M.M.: Efficient spatio-temporal tactile object recognition with randomized tiling convolutional networks in a hierarchical fusion strategy. In: Proc. of the 30th AAAI, pp. 3337-3345 (2016)

34. Gallace, A., Spence, C.: The cognitive and neural correlates of "tactile consciousness": A multisensory perspective. Consciousness and Cognition 17(1), 370-407 (2008)

35. Zeiler, M.D., Fergus, R.: Visualizing and understanding convolutional networks. In: Proc. of ECCV, pp. 818-833 (2014)

36. Ngiam, J., Chen, Z., Chia, D., Koh, P.W., Le, Q.V., Ng, A.Y.: Tiled convolutional neural nets. In: Proc. of NIPS, pp. 1279-1287 (2010)

37. Lee, H., Grosse, R., Ranganath, R., Ng, A.Y.: Convolutional deep belief networks for scalable unsupervised learning of hierarchical representations. In: Proc. of ICML, pp. 609-616 (2009)

38. Gong, Y., Wang, L., Guo, R., Lazebnik, S.: Multi-scale orderless pooling of deep convolutional activation features. In: Proc. of ECCV, pp. 392-407 (2014)

39. Chen, L.C., Papandreou, G., Kokkinos, I., Murphy, K., Yuille, A.L.: Deeplab: Semantic image segmentation with deep convolutional nets, atrous convolution, and fully connected CRFs. IEEE Transactions on Pattern Analysis and Machine Intelligence 40(4), 834-848 (2017)

40. Saxe, A., Koh, P.W., Chen, Z., Bhand, M., Suresh, B., $\mathrm{Ng}$, A.Y.: On random weights and unsupervised feature learning. In: Proc. of ICML, pp. 1089-1096 (2011)

41. Jarrett, K., Kavukcuoglu, K., Ranzato, M., LeCun, Y.: What is the best multi-stage architecture for object recognition? In: Proc. of CVPR, pp. 2146-2153 (2009)

42. Pinto, N., Doukhan, D., DiCarlo, J.J., Cox, D.D.: A high-throughput screening approach to discover good forms of biologically inspired visual representation. PLoS computational biology 5(11), e1000,579(1-12) (2009)

43. Huang, G.B., Bai, Z., Kasun, L.L.C., Vong, C.M.: Local receptive fields based extreme learning machine. IEEE Computational Intelligence Magazine 10(2), 18-29 (2015) 
44. He, K., Zhang, X., Ren, S., Sun, J.: Deep residual learning for image recognition. In: Proceedings of the IEEE conference on computer vision and pattern recognition, pp. 770-778 (2016)

45. Bicchi, A., Scilingo, E.P., Ricciardi, E., Pietrini, P.: Tactile flow explains haptic counterparts of common visual illusions. Brain research bulletin 75(6), 737-741 (2008)

46. Sun, D., Roth, S., Black, M.J.: Secrets of optical flow estimation and their principles. In: Proc. of CVPR, pp. 2432-2439 (2010)

47. Horn, B.K., Schunck, B.G.: Determining optical flow. Artificial Intelligence 17, 185-203 (1981)

48. Ioffe, S., Szegedy, C.: Batch normalization: Accelerating deep network training by reducing internal covariate shift. In: International Conference on Machine Learning, pp. 448-456 (2015)

49. Spratling, M.W.: A hierarchical predictive coding model of object recognition in natural images. Cognitive Computation 9(2), 151-167 (2017)

50. Szegedy, C., Liu, W., Jia, Y., Sermanet, P., Reed, S., Anguelov, D., Erhan, D., Vanhoucke, V., Rabinovich, A.: Going deeper with convolutions. In: Proc. of CVPR, pp. $1-9(2015)$

51. He, K., Zhang, X., Ren, S., Sun, J.: Spatial pyramid pooling in deep convolutional networks for visual recognition. In: Proc. of ECCV, pp. 346-361 (2014)

52. Hengshuang, Z., Jianping, S., Xiaojuan, Q., Xiaogang, W., Jiaya, J.: Pyramid scene parsing network. In: Proc. of CVPR, pp. 2881-2890 (2017)

53. Liu, X., Deng, Z.: Segmentation of drivable road using deep fully convolutional residual network with pyramid pooling. Cognitive Computation pp. 1-10 (2018)

54. Hu, X., Zhang, X., Liu, M., Chen, Y., Li, P., Pei, W., Zhang, C., Chen, H.: A flexible capacitive tactile sensor array with micro structure for robotic application. Science China Info. Sci. 57(12), 1-6 (2014)

55. Zhang, J., Cui, J., Lu, Y., Zhang, X., Hu, X.: A flexible capacitive tactile sensor for manipulator. In: Proc. of ICCSIP, pp. 303-309 (2016)

56. Nair, V., Hinton, G.E.: Rectified linear units improve restricted boltzmann machines. In: Proceedings of the 27th ICML, pp. 807-814 (2010)

57. Jia, Y., Shelhamer, E., Donahue, J., Karayev, S., Long, J., Girshick, R., Guadarrama, S., Darrell, T.: Caffe: Convolutional architecture for fast feature embedding. In: Proceedings of the 22nd ACM international conference on Multimedia, pp. 675-678. ACM (2014)

58. Scardapane, S., Wang, D.: Randomness in neural networks: an overview. Wiley Interdisciplinary Reviews: Data Mining and Knowledge Discovery 7(2), e1200 (2017)

59. Bo, L., Ren, X., Fox, D.: Hierarchical matching pursuit for image classification. In: Proc. of NIPS, pp. 2115-2123 (2011)

60. Saisan, P., Doretto, G., Wu, Y.N., Soatto, S.: Dynamic texture recognition. In: Proc. of CVPR, vol. 2, pp. 58-63 (2001)

61. Johnson, B.W.: Fault-tolerant microprocessor-based sys. IEEE Micro 4(6), 6-21 (1984)

62. Cao, L., Sun, F., Liu, X., Huang, W., Cheng, W., Kotagiri, R.: Fix-budget and recurrent data mining for online haptic perception. In: International Conference on Neural Information Processing, pp. 581-591 (2017) 